\title{
A life cycle alteration can correct molting defects in Caenorhabditis elegans
}

Shaonil Binti, Rosa V. Melinda, Braveen B. Joseph, Phil Edeen, Sam D. Miller, and David S. Fay*

Department of Molecular Biology, College of Agriculture and Natural Resources, University of Wyoming, Laramie, WY 82071

*Corresponding author 


\section{Abstract}

Molting is a widespread feature in the development of many invertebrates, including nematodes and arthropods. In Caenorhabditis elegans, the highly conserved protein kinases NEKL-2/NEK8/9 and NEKL-3/NEK6/7 (NEKLs) promote molting through their involvement in the uptake and intracellular trafficking of epidermal cargos. We found that the relative requirements for NEKL-2 and NEKL-3 differed at different life-cycle stages and under different environmental conditions. Most notably, the transition from the second to the third larval stage $(\mathrm{L} 2 \rightarrow \mathrm{L} 3 \mathrm{molt})$ required a higher level of NEKL function than during several other life stages or when animals had experienced starvation at the L1 stage. Specifically, larvae that entered the pre-dauer L2d stage could escape molting defects when transiting to the (non-dauer) L3 stage. Consistent with this, mutations that promote entry into $\mathrm{L} 2 \mathrm{~d}$ suppressed nekl-associated molting defects, whereas mutations that inhibit L2d entry reduced starvation-mediated suppression. We further showed that loss or reduction of NEKL functions led to defects in the transcription of cyclically expressed molting genes, many of which are under the control of systemic steroid hormone regulation. Moreover, the timing and severity of these transcriptional defects correlated closely with the strength of nekl alleles and with their stage of arrest. Interestingly, transit through $\mathrm{L} 2 \mathrm{~d}$ rescued nekl-associated expression defects in suppressed worms, providing an example of how life-cycle decisions can impact subsequent developmental events. Given that NEKLs are implicated in the uptake of sterols by the epidermis, we propose that loss of NEKLs leads to a physiological reduction in steroid-hormone signaling and consequent defects in the transcription of genes required for molting. 


\section{Introduction}

Developmental plasticity is critical for ensuring the survival of an organism in response to rapidly changing environments (Suzuki \& Nijhout, 2008). The dauer larva, a developmentally arrested stage of $C$. elegans, is a classic example of phenotypic plasticity that allows the worm to cope with the unpredictability of nature (Cassada \& Russell, 1975; Fielenbach \& Antebi, 2008; Golden \& Riddle, 1984b). Under permissive conditions after hatching from the egg, $C$. elegans develops through four larval stages (L1, L2, L3, and L4) and eventually becomes an adult (Figure 1A). Conversely, when the conditions are non-optimal (e.g., high temperature, crowding, limited food availability), late-stage L1 larvae sense the harsh environment and initiate a full-body response to form a dauer (also referred to as diapause) larva, an alternative form of L3 that is stress resistant, developmentally quiescent, and designed for survival and dispersion (Cassada \& Russell, 1975; Golden \& Riddle, 1984b). During the process of forming a dauer, the L1 larva first molts into a pre-dauer L2d larva Figure 1A). The decision to develop into either an L2 or L2d larva happens several hours before the $L 1 \rightarrow L 2$ (d) molt (Golden \& Riddle, 1984b; Schaedel et al., 2012). An L2d larva then has two options-either proceed to form a dauer or, if conditions improve, molt into an L3 larva and resume normal development. This decision is made at the mid-L2d stage, at which point the larva becomes committed to forming a dauer (L3d) or proceeding with L3 development (Figure 1A) (Schaedel et al., 2012). The initial decision to enter L2d from L1 provides a safety net, giving animals the ability to enter dauer $(\mathrm{L} 2 \mathrm{~d} \rightarrow \mathrm{L} 3 \mathrm{~d})$ should conditions fail to improve, while still allowing animals to resume with normal development $(L 2 d \rightarrow L 3)$ if the environment becomes permissive. One cost associated with $L 2 d$, however, is that that the $\mathrm{L} 2 \mathrm{~d}$ stage takes several hours longer to complete than $\mathrm{L} 2$, thus lengthening the time to become a fertile adult (Golden \& Riddle, 1984b; Karp, 2018). Other potential costs or benefits of the $L 2 d$ decision on subsequent developmental events are not well understood.

The endocrine signaling network that regulates the developmental switch between reproductive development and dauer formation has been extensively studied in C. elegans (Figure 1B). In well-fed animals, insulin/IGF-1-like peptides are secreted by neuroendocrine 
A

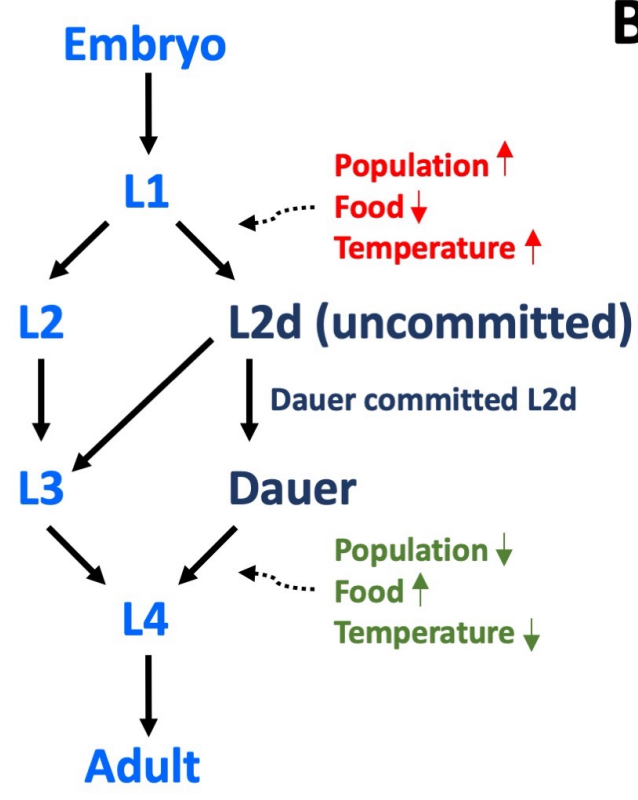

\section{Life cycle of $C$. elegans}

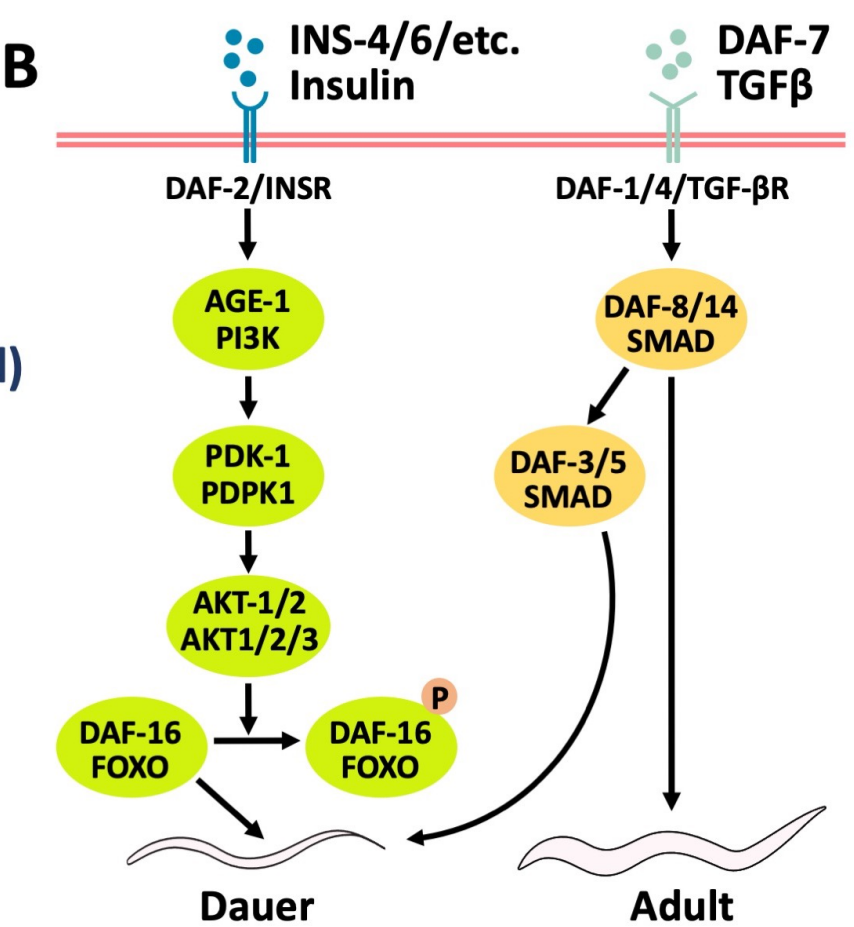

DAF-7 TGF $\beta$

Figure 1. Developmental trajectories of $C$. elegans. (A) Under ideal conditions, an L1 larva develops continuously through four larval stages $(L 1, L 2, L 3, L 4)$ to reach adulthood. Under unfavorable conditions (e.g., high temperature, high population density, or limited food availability), an L1 larva molts into an uncommitted $\mathrm{L} 2 \mathrm{~d}$ pre-dauer larva. If conditions improve, the L2d larva molts into an L3 and resumes reproductive development. Alternatively, if unfavorable conditions persist, the L2d becomes committed to forming a dauer (L3d), which exists in a state of arrested development until conditions improve, at which time it completes L3 programs and molts into an L4. (B) insulin/IGF-1 and TGF- $\beta$ signaling pathways integrate environmental cues, leading to appropriate developmental decisions. In the presence of food, insulin-like peptides bind to the DAF2/INSR receptor, which further activates the phospholipid kinase AGE-1/PI3K and the serine/threonine kinases PDK-1, AKT-1, and AKT-2. This signaling cascade leads to phosphorylation and nuclear exclusion of the DAF16/FOXO transcription factor, which is required for the upregulation of genes that promote dauer. Acting in parallel, the TGF- $\beta$ pathway is sensitive to changes in population density, food availability, and temperature. Under favorable conditions, TGF- $\beta$ signaling is initiated by the activation of DAF-1/4/TGF- $\beta$ R receptors by the DAF-7/TGF- $\beta$ ligand. This signal is transduced through the activation of DAF-8/SMAD2 and DAF-14/SMAD3, which in turn inhibit the positive regulators of dauer induction, DAF-3/SMAD4 and DAF-5/Ski/Sno.

cells and bind to DAF-2, the $C$. elegans homolog of the insulin/IGF-1 receptor $(\mathrm{Hu}, 2007$; Kimura et al., 1997). Ligand activation of DAF-2 further transduces the nutrient signal by activating downstream kinases that eventually phosphorylate and sequester the DAF-16/FOXO transcription factor in the cytoplasm (Lee et al., 2001; K. Lin, 1997; Kui Lin et al., 2001; Ogg et al., 1997). Reduced activity of the insulin/IGF-1 signaling pathway due to nutrient stress or mutations in upstream genes allows DAF-16 to enter the nucleus and promote the expression of target genes necessary for dauer transformation and arrest. Acting in a semi-parallel fashion to insulin/IGF-1, the TGF- $\beta$ signaling pathway is responsive to environmental cues such as dauer 
pheromone, which is an indicator of population density (Golden \& Riddle, 1984b; Ren et al., 1996; Riddle \& Albert, 1997). Under satisfactory conditions, secreted DAF-7/TGF- $\beta$ binds to the TGF- $\beta$ receptors DAF-1 and DAF-4, leading to the inhibition of downstream SMAD proteins DAF3/SMAD4 and DAF-5/Ski/Sno, which, like DAF-16, promote the dauer program (Fielenbach \& Antebi, 2008; Gumienny \& Savage-Dunn, 2013). The presence of dauer pheromone in the environment inhibits DAF-7 expression, thus allowing DAF-3 and DAF-5 to favor dauer formation. (Fielenbach \& Antebi, 2008; Ren et al., 1996). Acting together, these two pathways integrate diverse environmental cues, leading to an appropriate developmental choice.

Interestingly, worms do retain some memory of prior developmental choices. When compared with an adult that developed continuously, a post-dauer adult exhibits the differential expression of many genes including those associated with reproduction, transcriptional regulation, and metabolism (Hall et al., 2010). Dauer formation also suppresses abnormal germ cell proliferation in adults, suggesting that progression through dauer can alter phenotypes at subsequent life stages (Mao et al., 2020). Correspondingly, passage through dauer can correct spatial and temporal defects in cell fate patterning, indicating that dauer may induce compensatory mechanisms to maintain developmental robustness (Abrahante et al., 2003; Battu et al., 2003; Baugh \& Hu, 2020; Ferguson \& Horvitz, 1985; Ilbay \& Ambros, 2019).

In this study, we report the surprising finding that entry into uncommitted $L 2 d$ can compensate for a defect in the $C$. elegans molting cycle. Molting is a relatively understudied but crucial developmental process that allows $C$. elegans to grow and progress through larval stages into an adult (Lažetić \& Fay, 2017b). During each molt, the old cuticle detaches from the underlying epidermis, while a new cuticle is synthesized underneath. Molting is orchestrated in large part by nuclear hormone receptor signaling, which controls the expression of thousands of genes that oscillate with the molting cycle (Antebi, 2015; Frand et al., 2005; Hendriks et al., 2014; Meeuse et al., 2020; Turek \& Bringmann, 2014). One key molecule in this process is cholesterol obtained from the environment, which is required for the synthesis of steroid hormone ligands that activate molting-associated nuclear hormone receptors such as NHR-23/RORC and NHR- 
25/NR5A1/2 (Asahina et al., 2000; Kostrouchova et al., 2001; Kurzchalia \& Ward, 2003; Lažetić \& Fay, 2017b; Monsalve \& Frand, 2012). Consistent with this, C. elegans requires dietary cholesterol for progression through all four larval stages, and cholesterol deprivation impairs growth and other physiological processes including molting (Shim et al., 2002; Yochem et al., 1999). The importance of steroid hormone signaling is also underscored by the observation that the epidermally expressed low-density lipoprotein (LDL)-like receptor LRP-1/Megalin is required for molting and is thought to mediate the uptake of sterols by the epidermis.

We previously showed that the NIMA-related kinases NEKL-2/NEK8/9 and NEKL-3/NEK6/7 (NEKLs)-along with their conserved ankyrin repeat binding partners, MLT-2/ANKS6, MLT3/ANKS3, and MLT-4/INVS (MLTs) - promote molting through their roles in regulating the trafficking of epidermal cargos. In larvae harboring mutations in nekl and $m / t$ genes, the old cuticle fails to detach fully during molting and worms undergo larval arrest (Lažetić \& Fay, 2017a; Yochem et al., 2015). Within the epidermis, depletion of NEKLs leads to impaired clathrin-mediated endocytosis and reduced internalization of LRP-1 (Joseph et al., 2020). Here we show that downregulation of NEKLs results in the delayed and/or reduced expression of hormonally regulated molting-specific genes, thereby establishing a link between NEKL trafficking functions and the expression of genes required for molting. Notably, this defect can in some circumstances be overcome by entry into $L 2 d$, which, together with other data, indicates clear differences in the requirements for NEKLs at different molts along with demonstrating the impact of life-cycle decisions on downstream phenotypic outcomes.

\section{Materials and Methods}

\section{Strains and maintenance}

C. elegans strains were cultured on nematode growth medium (NGM) spotted with E. coli OP50 and maintained at $20^{\circ} \mathrm{C}$, unless noted otherwise. Synchronous populations of L1 larvae were obtained by bleaching gravid adults (Figures 7A, 8, and S4-S7; Porta-de-la-Riva et al., 2012). After the bleach treatment, eggs were washed five times and allowed to hatch in M9 buffer overnight at room temperature. Hatched worms remain arrested as L1 larvae in the M9 buffer 
until they are transferred to a plate containing food. All strains used in this study are listed in File S1.

\section{CRISPR/Cas9-generated loss-of-function mutation in daf-16}

The $f d 335$ allele of $d a f-16$ was created using the $d p y$-10 co-CRISPR approach (Arribere et al., 2014; Kim et al., 2014; Paix et al., 2014, 2015). The $f d 335$ allele contains two premature stop codons at (K248Stop and G250Stop) in DAF-16a, along with an introduced restriction site. Primer sequences for the sgRNA and repair template are given below. Capital letters represent the altered or inserted nucleotides.

sgRNA for daf-16(fd335): gtacgccgtggattccttcc

Repair Template:

attcagaatgaaggagccggaaagagctcgtggtgggttattaatccagaAgcTTagccaTgaaggaatcacggcgtacacgtgaac gatccaatactattgagacgactacaaaggtaagaga nekl-2(fd81); daf-16(fd335); nekl-3(gk894345); fdEx286 worms were allowed to starve and were subsequently examined for the presence of dauers. We failed to detect $\left(\mathrm{GFP}^{+}\right)$dauer worms based on morphology. In addition, we failed to detect any surviving worms following treatment with $1 \%$ SDS, further indicating that $f d 335$ blocks dauer formation.

\section{Image acquisition}

For the auxin treatment experiments in Figure S3, fluorescence images were obtained using an Olympus IX83 P2ZF inverted microscope. Z-stacks were captured with a step size of $0.2 \mu \mathrm{m}$, using a 100× silicon oil immersion objective (1.35 N.A.). Images in Figures S5 and S6 were taken with an Olympus MVX10 MacroView microscope using a $2 \times$ objective (0.5 N.A.). The rest of the DIC images and fluorescence images were obtained using a Nikon Eclipse epifluorescence microscope using $10 \times(0.25$ N.A.) and $40 \times(0.75$ N.A.) objectives. Images were acquired using Olympus cellSens software version 3.1. Before imaging, animals were mounted on 3\% agarose pads and immobilized using $10 \mathrm{mM}$ levamisole. 


\section{Image analysis}

All the image processing and analyses were performed using FIJl software (Schindelin et al., 2012). For the auxin treatment experiments, the fluorescence images were first background subtracted, and then image thresholding was performed using the Li algorithm (Li \& Tam, 1998). A representative region of interest in hyp7 was selected that covered most of hyp7 in the frame. Then the percentage of positive pixels was quantified for the selected region of interest.

\section{Auxin-mediated degradation studies}

The protocol for the auxin treatment experiments was performed as described in our previous paper (Joseph et al., 2020). To generate the graphs on the left in Figure 6C-E, development was synchronized by bleaching (Porta-de-la-Riva et al., 2012), except for the synchronization of L3 and L4 stages of nekl-3::aid genotype which was done manually by characterizing vulval and gonad development. Animals were treated with auxin during the early period of larval development at L1, L2, L3, and L4 stages. Identification of larval stages was performed by following gonad morphology under the dissecting scope. To generate the graphs on the right in Figure $6 \mathrm{C}-\mathrm{E}$, starved worms were gently washed off the plate and treated with $1 \%$ SDS to isolate dauer larvae (Karp, 2018). Isolated dauers were transferred to an unseeded plate (i.e., one without a bacterial lawn) and treated with auxin for 20 hours. After the auxin treatment, dauers were transferred back to a seeded plate (without auxin) to allow them to grow.

For the experiments in Figure S3, all three strains (wild type, nekl-3::aid, and daf-2; nekl-3::aid) were cultured at $22^{\circ} \mathrm{C}$ until daf-2; nekl-3::aid mutants formed dauers. Dauer larvae were identified based on morphological characteristics and transferred to a separate plate. Strains were then incubated at $16^{\circ} \mathrm{C}$ until daf-2; nekl-3::aid dauers molted into L4s. From these plates, L4 larvae were selected and transferred to separate plates where they continued to develop into adults. Day-1 adults of wild type, nekl-3::aid, and daf-2; nekl-3::aid genotypes were treated with auxin for 20 hours before imaging. For each experiment, at least 30 worms were imaged for the expression of LRP-1::GFP in the hyp7 epidermis. 


\section{Dauer pheromone experiments}

For dauer pheromone experiments, NGM plates were made without peptone. Synthetic ascarosides (ascr\#2, ascr\#3, and ascr\#5), which were kindly provided by the Schroeder lab (Butcher et al., 2008), were added to the liquid medium before it was poured into the plates, so that the final concentration of each ascaroside was $3 \mu \mathrm{M}$ (Ilbay \& Ambros, 2019). Poured plates were spotted with heat-killed $E$. coli OP50. To prepare the bacteria, $5 \mathrm{~mL}$ of an overnight culture of Escherichia coli OP50 was pelleted, washed twice with sterile water, and dissolved in a tube containing $1 \mathrm{~mL}$ of sterile water. The tube was then placed in a water bath set at $95^{\circ} \mathrm{C}$ for $30 \mathrm{~min}$ and vortexed vigorously every $5 \mathrm{~min}$ during this incubation (Hollister et al., 2013). Six gravid adults were placed onto an unseeded plate to clear off excess live $E$. coli. They were then transferred to dauer pheromone-containing plates and allowed to lay eggs for 4 hours before being removed. Worms were grown on dauer pheromone plates until nekl-2; nekl-3 (GFP ${ }^{+}$) worms formed dauers. Larvae were then washed off the plates with M9 and transferred to fresh NGM plates spotted with live E. coli. nekl-2; nekl-3 (GFP-) worms were scored for molting defects after $\sim 48$ hours.

\section{Pharyngeal pumping assay}

Gravid adults were treated with bleach to generate synchronized populations of L1 larvae as described above. Pharyngeal pumping was scored for $>50$ synchronized larvae every hour at $20^{\circ} \mathrm{C}$ after release from L1 arrest. An Olympus MVX10 MacroView microscope was used with a $2 \times$ objective to observe and score pharyngeal pumping of worms. Additional information is in File S2.

\section{Transcriptional analysis of molting genes}

Synchronized L1 larvae (obtained as described above) were cultured at $20^{\circ} \mathrm{C}$ after release from L1 arrest. Ten worms were scored for the expression of reporter genes every hour using the Olympus MVX10 MacroView microscope with a 2x objective. Worms were scored as positive or negative for marker expression, leading to the determination of the percentage of worms 
expressing (\% expression) used in Figures 8 and S4. Reduced/inconsistent expression was still scored as positive for the marker expression.

\section{Statistical Analysis}

GraphPad Prism 9 software was used to perform all statistical analyses and to generate bar graphs and line plots. 


\section{Results}

\section{Starvation as a suppressor of nekl-2; nekl-3 molting defects}

We previously identified weak loss-of-function alleles of nekl-2(fd81) and nekl-3(gk894345) that do not exhibit molting defects on their own but, when combined, exhibit synthetic lethality with $\sim 98.5 \%$ of worms arresting with molting defects (Figure 2A) (Lažetić \& Fay, 2017a). Most typically, nekl-2(fd81); nekl-3(gk894345) (hereafter nekl-2; nekl-3) mutants transit the L1 $\rightarrow$ L2 molt but then arrest at the $\mathrm{L} 2 \rightarrow \mathrm{L} 3$ molting stage. Arrested worms exhibit a partial cuticleshedding defect (the "corset" phenotype), in which the old cuticle fails to detach from the midbody region (Yochem et al., 2015). nekl-2; nekl-3 double mutants are maintained by the presence of an extrachromosomal array (fdEx286) that expresses wild-type nekl-3 along with a broadly expressed GFP reporter (Figure 2A).

In our studies, we observed that starved plates containing nekl-2; nekl-3 larvae produced elevated levels of array-minus $\left(\mathrm{GFP}^{-}\right)$adults following transfer to plates containing bacterial food. To quantify this phenomenon and to determine if starvation length is a factor in suppression, we first assayed non-synchronized worm populations from plates that had been starved for $0,1,3$, or 7 days prior to food reintroduction (Figure 2B). Notably, starvation of nekl2; nekl-3 double mutants for 1, 3, or 7 days led to a highly significant increase in the percentage of nekl-2; nekl-3 (GFP') animals that reached adulthood as compared with the absence of starvation (Figure 2B). Furthermore, we observed no significant differences in nekl-2; nekl-3 suppression between plates that had been starved for 1, 3, or 7 days, indicating that the suppressive effects of starvation occur within 24 hours (Figure 2B).

\section{Environmentally induced L2d can alleviate molting defects in nekl-2; nekl-3 mutants}

To better define the crucial developmental window for starvation-mediated suppression, we synchronized newly hatched nekl-2; nekl-3 L1 larvae and then transferred them to plates containing food for varying amounts of time. Larvae were then washed and moved to plates lacking food for 48 hours to induce a starvation response. Finally, larvae were returned to 
A
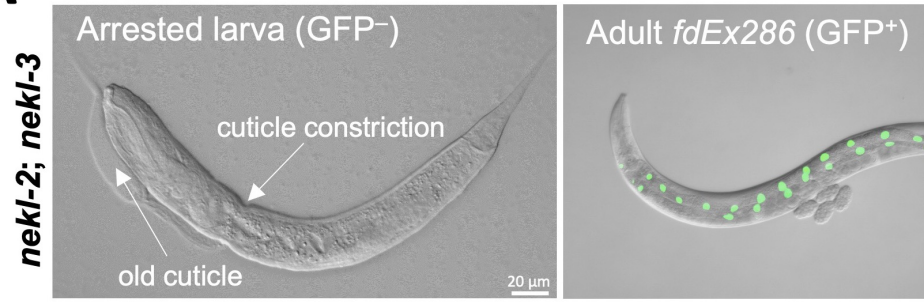

B
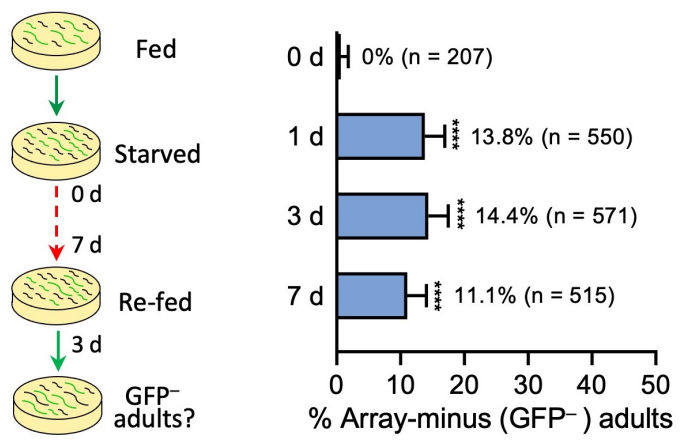

C
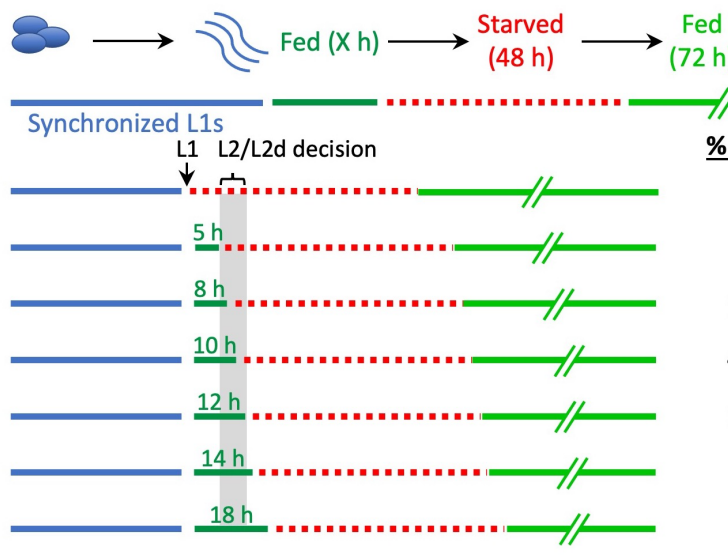

\section{in}

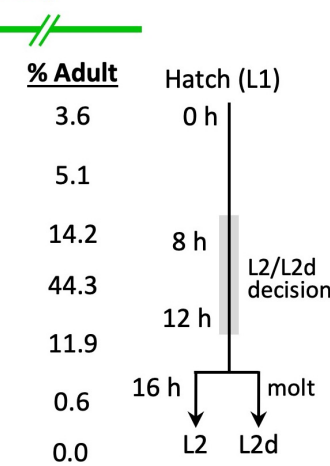

Figure 2. Starvation-induced suppression of molting defects in nekl-2; nekl-3 mutants.

(A) Representative images (DIC merged with green fluorescence) of nekl-2; nekl-3 double mutants without (left) and with (right) the rescuing extrachromosomal array (fdEx286), which encodes wild-type NEKL-3 and the SUR$5::$ GFP reporter. White arrows indicate the position of the constriction where the old cuticle failed to detach from the epidermis (double cuticle) and a portion of the old anterior cuticle that is still partially attached to the worm. (B) Mixed-stage populations of nekl-2; nekl-3 double mutants were starved for $0,1,3$, or 7 days. The number of viable array-minus $\left(\mathrm{GFP}^{-}\right)$adults and array-positive $\left(\mathrm{GFP}^{+}\right)$adults were counted after worms were transferred to a plate containing food and allowed to grow for 3 days. Bar graph represents the percentage of array-minus $\left(\mathrm{GFP}^{-}\right)$adults observed in the total adult population $\left(\mathrm{GFP}^{+}+\mathrm{GFP}^{-}\right)$. The percentage of nekl-2; nekl-3 (GFP') viable adults after 1, 3, and 7 days of starvation was statistically significant as compared with 0 days $(* * * * p<$ 0.0001 as determined by Fisher's exact test). The total number of adult worms is shown in parentheses after each percentage. (C) Synchronized nekl-2; nekl-3 L1 larvae were fed for variable lengths of time ( $X$ hours) before being starved for 48 hours and then returned to plates containing food for 72 hours. The percentage of viable GFP $^{-}$adults, [GFP ${ }^{-}$adults $\div\left(\mathrm{GFP}^{-}\right.$adults $+\mathrm{GFP}^{-}$arrested larvae $\left.) \times 100\right]$, was then scored. A corresponding timeline showing the period of the $L 2 \rightarrow L 2 d$ decision is shown to the right.

plates containing food for 72 hours to allow for the growth of suppressed worms to adulthood (Figure 2C). Interestingly, we found that induction of a starvation response, after the larvae were first grown for 10 hours on food, led to the highest levels of suppression, whereas starvation after 8 or 12 hours on fed plates led to moderate levels of suppression (Figure 2C). In contrast, starvation after growth on food for 14 or 18 hours failed to induce any suppression, and starvation directly after hatching ( 0 hours) or after 5 hours on food led to only low levels of suppression (Figure 2C). Notably, the optimal period for inducing suppression corresponded with the known time point at which L1 larvae become committed to either the L2 or L2d 
developmental programs (Golden \& Riddle, 1984b). Moreover, larvae that experienced starvation after the $\mathrm{L} 2 \rightarrow \mathrm{L} 2 \mathrm{~d}$ decision point (e.g., 14 and 18 hours) were unable to enter $\mathrm{L} 2 \mathrm{~d}$, consistent with the absence of suppression. Taken together, our findings suggest that progression through L2d, but not starvation per se, can alleviate molting defects in nekl-2; nekl3 worms. Moreover, the ability of the L1-specific starvation regimen to reduce nekl-2; nekl-3 molting defects indicates that uncommitted L2d, but not committed L2d or dauer entry, is sufficient for suppression.

To further test if the L2d developmental trajectory can suppress nekl molting defects, we used a mixture of ascarosides (collectively known as dauer pheromone) that induce a high percentage of $L 2 d$ and dauers in wild type (Butcher et al., 2008). We found that $~ 17 \%$ of the nekl-2; nekl-3 double mutants were able to reach adulthood when briefly exposed to ascarosides (Figure 3A), consistent with the model that nekl-2; nekl-3 mutants can be suppressed by transit through L2d. Furthermore, two findings confirmed that the conditions used here were effective at triggering progression through L2d/dauer. (1) Treatment of worms carrying a validated marker

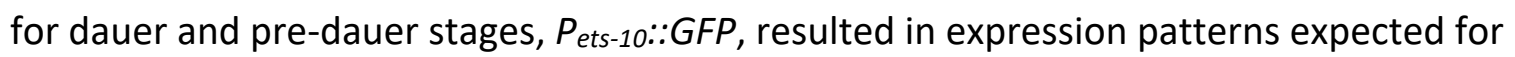
uncommitted L2d, committed L2d, and dauer stages (Figures 3B and S1; Shih et al., 2019). (2) nekl-2; nekl-3 double mutants carrying the nekl-3+; GFP+ rescuing array readily formed dauers in response to ascarosides as well as starvation. We note, however, that (GFP') nekl-2; nekl-3 double mutants uniformly failed to form dauers when subjected to starvation ( $n>1000)$, indicating that nekl-2; nekl-3 double mutants are at least partially defective in the $\mathrm{L} 2 \mathrm{~d} \rightarrow$ dauer transition. Curiously, when treated with ascarosides, $\sim 70 \%$ of nekl-2; nekl-3 mutants ( $n=228$ ) were able to form dauers, suggesting that the strength or method of the dauer induction can impact the ability of nekl-2; nekl-3 mutants to execute the $\mathrm{L} 2 \mathrm{~d} \rightarrow$ dauer transition.

To further strengthen the link between L2d and the suppression of molting, we crossed the $P_{\text {ets- }}$ 10::GFP marker into nekl-2; nekl-3 mutants. Late-stage L1 or early-stage L2 nekl-2; nekl-3 larvae were identified from a population that had experienced starvation for 1 day. We next sorted 
A

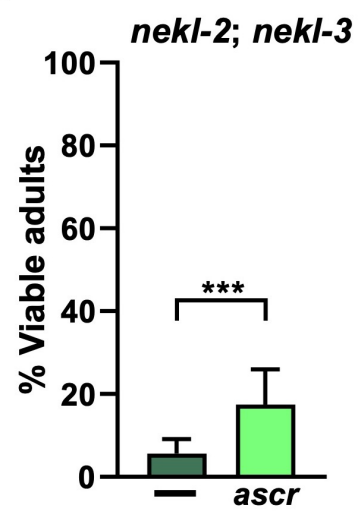

C

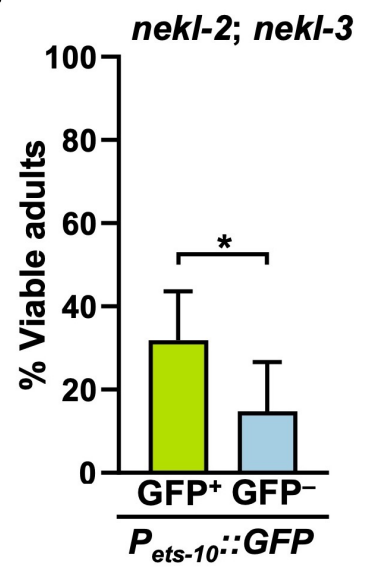

B

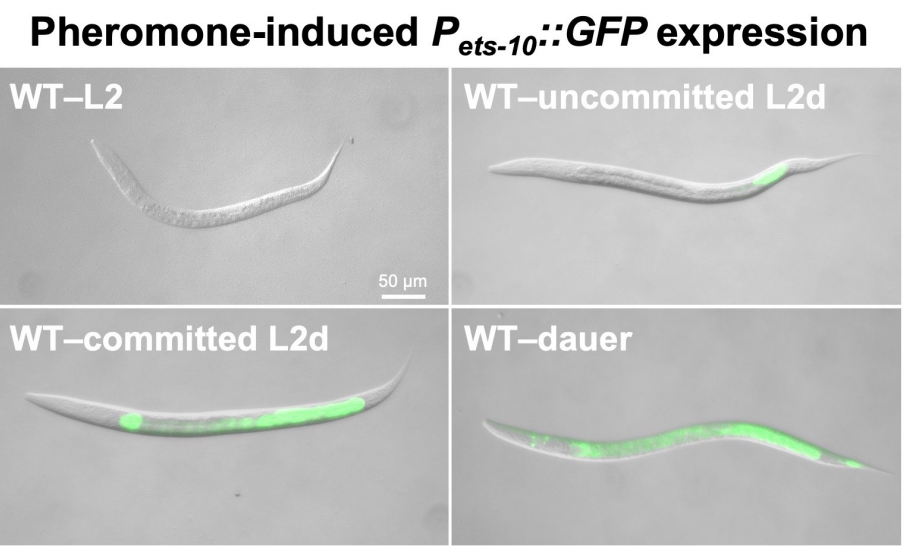

D

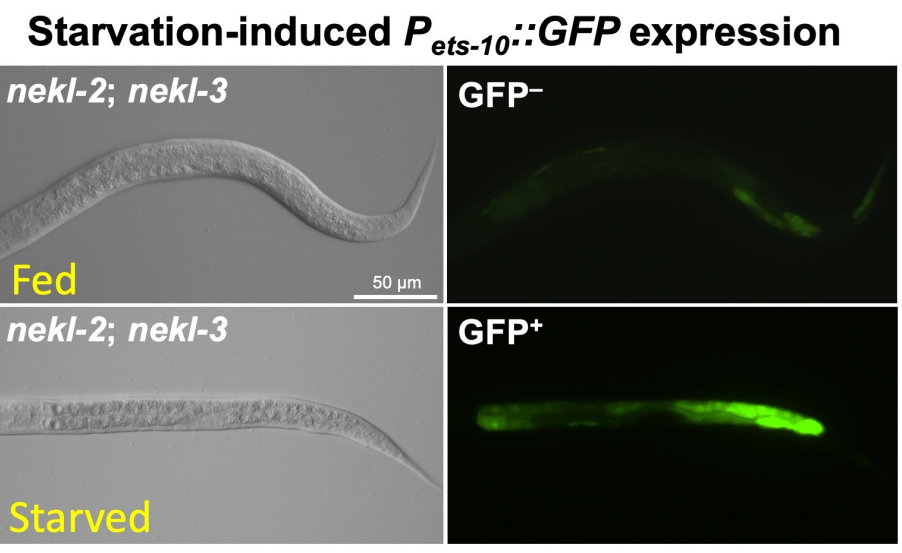

Figure 3. L2d induced by dauer pheromone or starvation suppresses molting defects. (A) Graph showing the percentage of viable nekl-2; nekl-3 adults in the absence and presence of dauer-promoting ascarosides (ascr). (B)

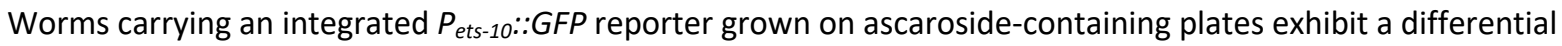
pattern of expression during dauer formation. Expression of $P_{\text {ets-10 }}:$ GFP was limited to the posterior intestine of uncommitted $\mathrm{L} 2 \mathrm{~d}$ larvae but then spread throughout the intestine as $\mathrm{L} 2 \mathrm{~d}$ larvae became committed to forming dauers. (C) Late-stage L1 and early-stage L2 nekl-2; nekl-3 larvae were starved for 1 day, transferred to food

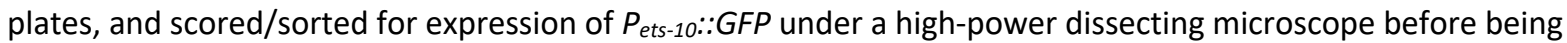
transferred to plates with bacteria. Worms were then assessed several days later for progression to adulthood. (D) Representative images of nekl-2; nekl-3 mutants (roughly staged as late L1 to L2) expressing $P_{\text {ets-10::GFP under }}$ well-fed (upper) and starved (lower) conditions. Error bars represent $95 \%$ confidence intervals. All statistical tests were performed using Fisher's exact test; * $p<0.05, * * * p<0.001$.

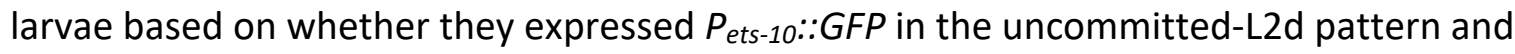
categorized the larvae as $\mathrm{GFP}^{+}$or $\mathrm{GFP}^{-}$before being moved to plates spotted with live $E$. coli (Figure 3D). Notably, starved GFP+ larvae were more likely to mature into adults as compared with their GFP- siblings (Figure 3C). This difference was significant even though it is likely that some of the GFP- worms had entered L2d but that the marker was too dim to be detected by 
our methods. Taken together, our findings indicate that L2d larvae have a higher tendency to overcome nekl molting defects and reach adulthood as compared with L2 larvae.

\section{Dauer-constitutive mutations alleviate nekl molting defects}

We next determined if the dauer-inducing signals of dauer-constitutive (Daf-c) mutations could also suppress molting defects in nekl-2; nekl-3 mutants. Downregulation of DAF-2/IGF1R and DAF-7/TGF- $\beta$ promote dauer entry by de-repressing downstream dauer-promoting transcription factors (Figure 1B). As such, loss-of-function mutations in daf-2 and daf-7 lead to worms forming dauers even in the presence of ample food (Gems et al., 1998; Karp, 2018; Riddle \& Albert, 1997). Because dauer formation is a temperature-sensitive process (Golden \& Riddle, 1984a), we scored for suppression of molting defects by daf-2 at two different temperatures, $16^{\circ} \mathrm{C}$ and $20^{\circ} \mathrm{C}$. Loss of function of daf-2 resulted in $\sim 30 \%$ of nekl-2; nekl-3 mutants reaching adulthood at $20^{\circ} \mathrm{C}$, whereas no suppression was observed at $16^{\circ} \mathrm{C}$ (Figure $4 A-B)$. Likewise, we observed robust suppression ( $\sim 60 \%$ ) of molting defects by daf-7 at $20^{\circ} \mathrm{C}$ (Figure 4B-C). We note that because nekl-2(fd81) is itself a temperature-sensitive allele, we were unable to perform suppression assays at $25^{\circ} \mathrm{C}$ (also see below). Furthermore, consistent with our prior observations under starvation conditions, the large majority of nekl-2; daf-2; nekl-3 mutants failed to form mature dauers, indicating a requirement for NEKLs in the $\mathrm{L} 2 \mathrm{~d} \rightarrow$ dauer transition. Together, these results suggest that triple mutants entered $\mathrm{L} 2 \mathrm{~d}$ at $20^{\circ} \mathrm{C}$ but then resumed reproductive development and that progression through $L 2 d \rightarrow L 3$ was sufficient to alleviate molting defects.

Given our previous data showing that starvation-induced L2d can suppress molting defects in nekl-2; nekl-3 mutants, we were curious to determine whether mutations that prevent dauer formation would also prevent suppression by starvation. To test this, we assayed nekl-2; nekl-3 suppression by starvation in daf-16, daf-5, and daf-16; daf-5 dauer-defective (Daf-d) backgrounds. Interestingly, although the CRISPR-generated allele daf-16(fd335) prevented the induction of dauer (see Materials and Methods), it resulted in only a minor reduction in 


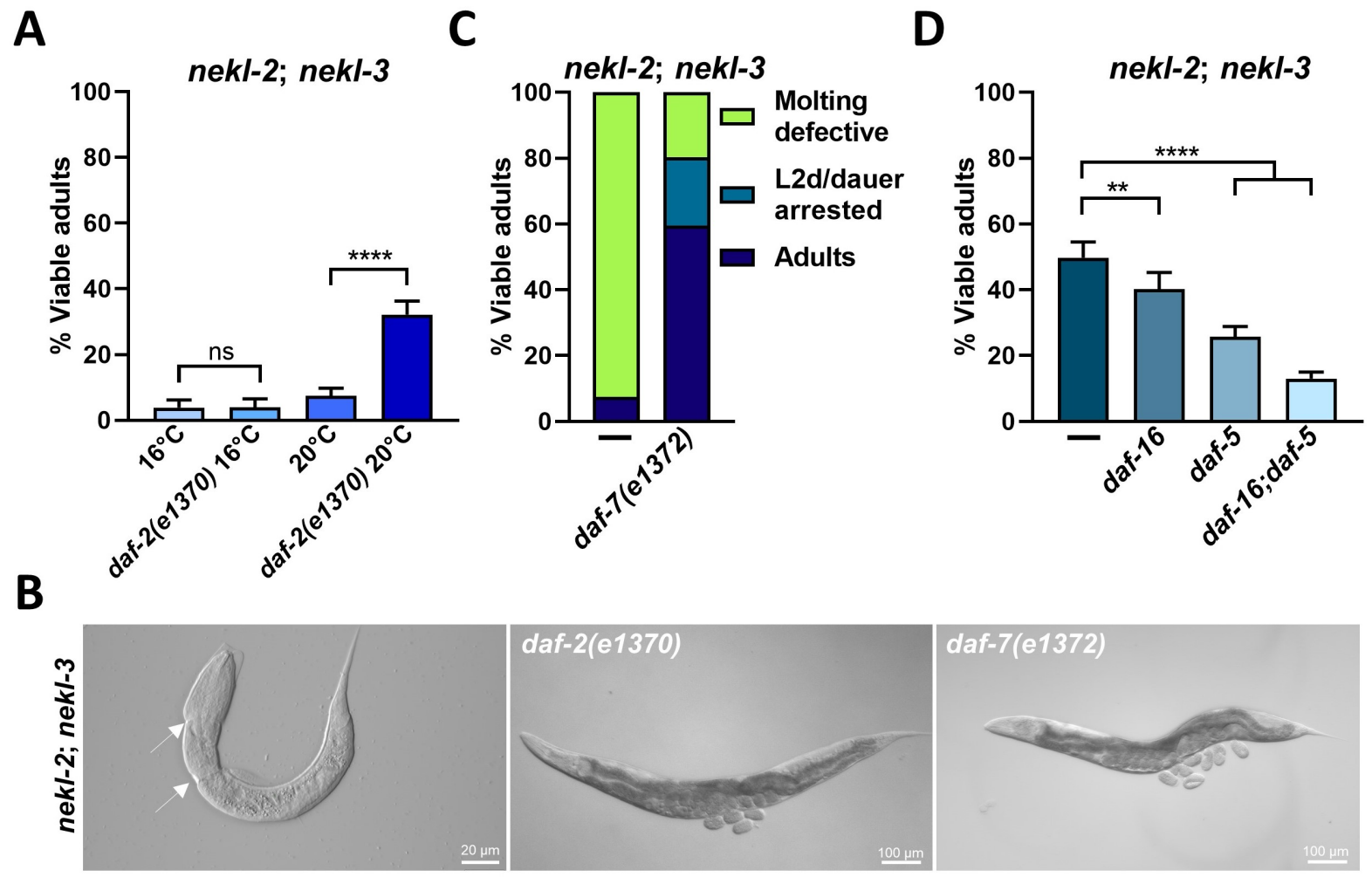

Figure 4. Genetic analysis is consistent with L2d-mediated suppression of molting defects. (A) Bar graph showing the percentage of viable adults when nekl-2; nekl-3 or nekl-2; daf-2; nekl-3 mutants were grown at $16^{\circ} \mathrm{C}$ or $20^{\circ} \mathrm{C}$. (B) DIC images of the mutants assessed in panel $\mathrm{A}$ at $20^{\circ} \mathrm{C}$. White arrows show region(s) of constriction where the old cuticle has failed to detach from the epidermis. (C) Graph showing viable adults (\%) among nekl-2; nekl-3 animals in the absence or presence of the daf-7 mutation. As strains containing daf-7(e1372) form dauers at $16^{\circ} \mathrm{C}$, we scored suppression at $20^{\circ} \mathrm{C}$ only. (D) nekl-2; nekl-3 mutants in the presence of Daf-d mutations daf-5 and daf16 , were allowed to starve for three days. Then strains were chunked to new plates and the percentage of viable adults was scored after $\sim 48$ hours. Error bars represent $95 \%$ confidence intervals. Statistical significance was determined using Fisher's exact test; $* * p<0.01, * * * * p 0.0001$. ns, not significant.

suppression by starvation in the nekl-2; nekl-3 background (Figure 4D). Consistent with this, suppression of nekl-2; nekl-3 by the Daf-c allele daf-2(e1370) was not significantly reversed by the Daf-d daf-16(fd335) mutation (Figure S2). In the case of daf-5, we observed a reduction in starvation-induced suppression by $\sim 2$-fold, whereas the daf-16; daf-5 combination reduced suppression by $\sim 5$-fold. Thus, whereas Daf-d mutations reduced the effectiveness of starvation-mediated suppression to varying extents, they did not entirely prevent it. This may be because these mutations, while fully preventing dauer formation, do not completely prevent entry into uncommitted-L2d. Collectively, our genetic data support a model of nekl-2; nekl-3 suppression that relies on entry into uncommitted L2d and suggest that Daf-d mutations may retain some ability to enter $L 2 d$ or a partial L2d-like state. 


\section{The $\mathbf{L} \mathbf{2 d} \rightarrow \mathbf{L} 3$ developmental trajectory is not sufficient to suppress strong loss-of-function nekl mutations}

We next determined whether starvation can suppress developmental arrest in other nekl and $m / t$ alleles that exhibit molting defects at either the $\mathrm{L} 1 \rightarrow \mathrm{L} 2$ or $\mathrm{L} 2 \rightarrow \mathrm{L} 3$ transitions. We did not observe any suppression of $\mathrm{L} 1 \rightarrow \mathrm{L} 2$ molting defects in strong or null alleles of nekl-2, nekl-3, and $m / t-3$ (Figure 5A). This result was expected, as mutant larvae become arrested prior to entering L2d. Interestingly, we also observed no suppression of molting defects in intermediate loss-offunction nekl and $m / t$ backgrounds that cause arrest at $\mathrm{L} 2 \rightarrow \mathrm{L3}$ [nekl-2(fd91), nekl-3(sv3), and $m / t-4(s v 9)$ ] (Figure 5A), indicating that suppression by L2d may be allele specific.

As a further test, we examined the ability of mutations in daf- 2 and $d a f-7$ to suppress $L 2 \rightarrow L 3$ molting defects in nekl-2(fd91) and nekl-3(sv3) intermediate loss-of-function mutants. Both daf2 and $d a f-7$ led to weak, but above background, suppression of nekl-2(fd91) but failed to suppress molting defects in nekl-3(sv3) mutants (Figures $5 \mathrm{~B}-\mathrm{C}$ ), which may cause more severe molting defects than $f d 91$ (Lažetić \& Fay, 2017a; Yochem et al., 2015). One potential explanation for the relative lack of suppression of nekl-2(fd91) and nekl-3(sv3) mutants by starvation or mutations is that they are defective at entry into $\mathrm{L} 2 \mathrm{~d}$. To determine whether this

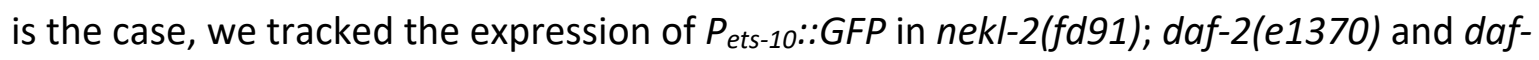
2(e1370); nekl-3(sv3) larvae. Notably, we observed L2d-like expression of $P_{\text {ets-10::GFP in } 100 \% \text { of }}$ second-stage larvae harboring the Daf-c mutations (Figure 5D). Collectively, our results indicate that the stage at which arrest occurs, although a factor, is not the only criterion required for L2d-mediated suppression. Rather, these data suggest that $L 2 d$ can induce suppression when NEKL and MLT function has not been compromised beyond a critical threshold. 
A

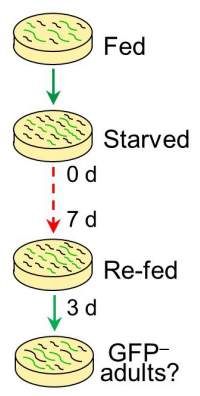

nekl-2(fd90)

nekl-2(gk839)

nekl-3(gk506)

$m / t-3(f d 72)$

nekl-2(fd91)

$n e k l-3(s v 3)$

$m / t-4(s v 9)$

B

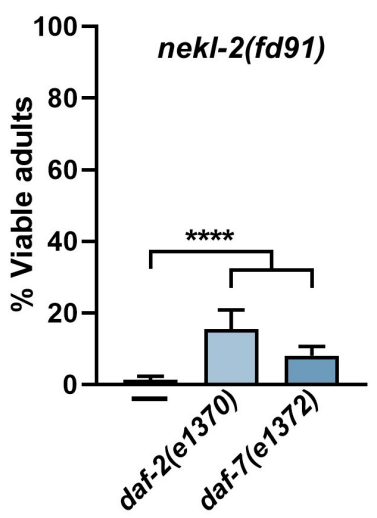

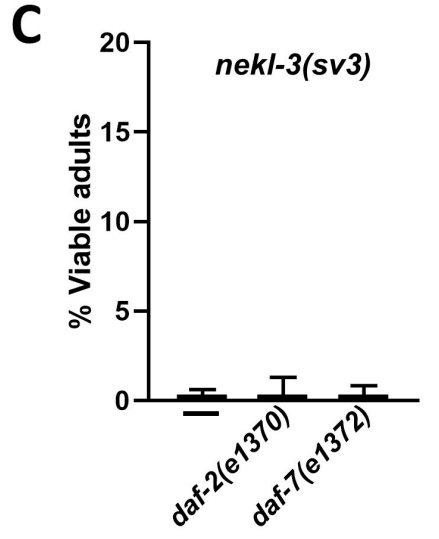

$P_{\text {ets-10: :GFP expression }}$

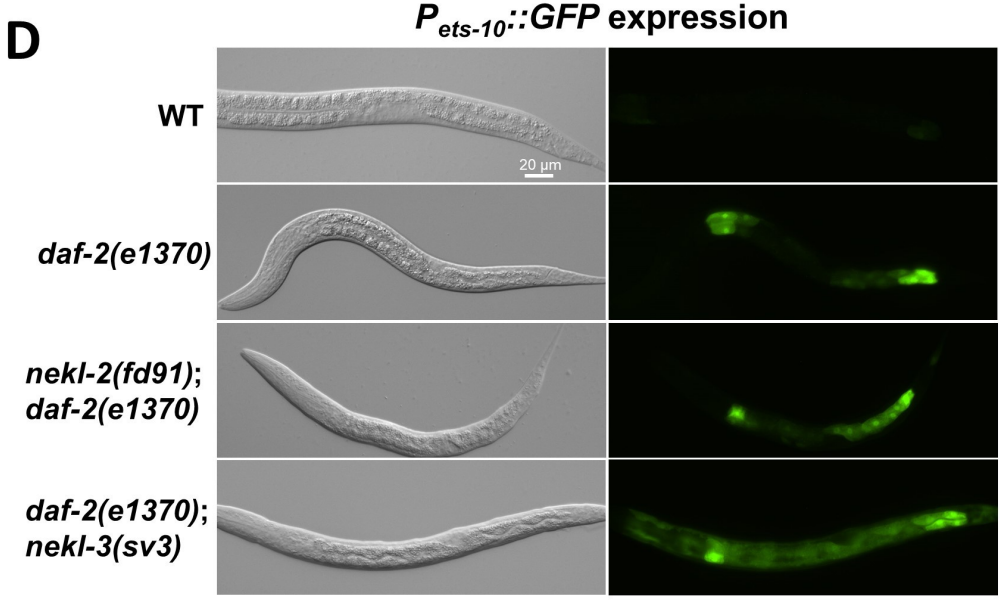

Figure 5. Transit through L2d does not suppress strong loss-of-function alleles of nekl-2 or nekl-3. (A) Mixed-stage populations of null [nekl-2(fd90), nekl2(gk839), nekl-3(gk506), and $m / t-3(f d 72)]$ and intermediate loss-of-function [nekl2(fd91), nekl-3(sv3), and $m / t-4(s v 9)]$ mutants were starved for $0,1,3$, and 7 days, and the percentage of viable arrayminus (GFP-) adults was scored as described in Figure 2B. The total number of worms is shown in parentheses after each percentage (ND, not determined.) (B, C) Bar graphs showing the percentage of viable adults in nekl-2 (B) and nekl-3 (C) intermediate loss-of-function backgrounds containing the daf-2 or daf-7 Daf-c mutations. (D) Representative images of $L 2$ or L2d larvae expressing the $P_{\text {ets-10::GFP reporter in the assayed }}$ backgrounds. Ten or more L2-stage worms were scored for the expression of the $P_{\text {ets-10::GFP reporter under a }}$ compound fluorescence microscope. Error bars represent $95 \%$ confidence intervals. Statistical significance was determined using Fisher's exact test; $* * * * p<0.0001$.

Different molting stages have different requirements for NEKLs The ability of nekl-2; nekl-3

double mutants to transit the $\mathrm{L} 2 \mathrm{~d} \rightarrow \mathrm{L} 3$ molt but not the $\mathrm{L} 2 \rightarrow \mathrm{L} 3$ molt led us to hypothesize that the requirements for NEKL-2 and NEKL-3 may vary between different molts. To investigate the requirement for NEKL kinases at different larval-stage molts, we used the auxin-inducible degradation (AID) system to rapidly deplete CRISPR-tagged nekl-2::aid and nekl-3::aid strains at 
specific time points close to the start of each larval stage (Figure 6A) (Holland et al., 2012;

Joseph et al., 2020; Zhang et al., 2015). In the case of NEKL-2, we observed substantial molting defects in all subsequent molts, indicating that NEKL-2 is required for all four molts during continuous development (Figures 6C). Unlike NEKL-2, depletion of NEKL-3 led to 100\% molting defects only during the $\mathrm{L} 1 \rightarrow \mathrm{L} 2$ and $\mathrm{L} 2 \rightarrow \mathrm{L} 3$ molts, but only a small population of worms

A

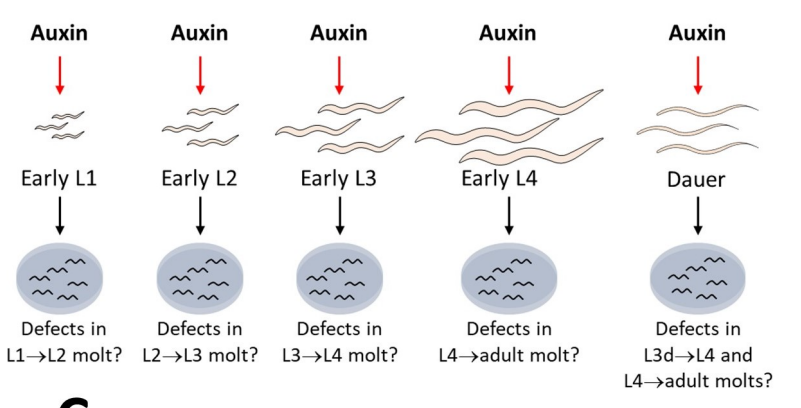

B

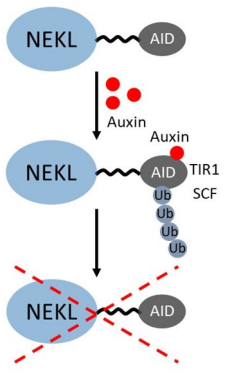

C nekl-2::aid

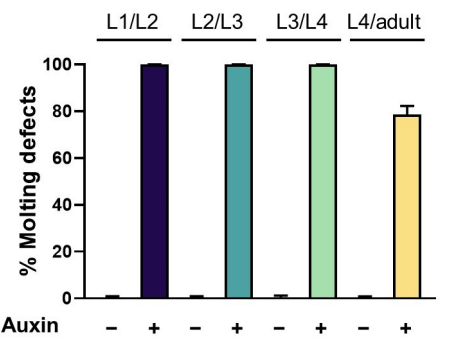

D

nekl-3::aid

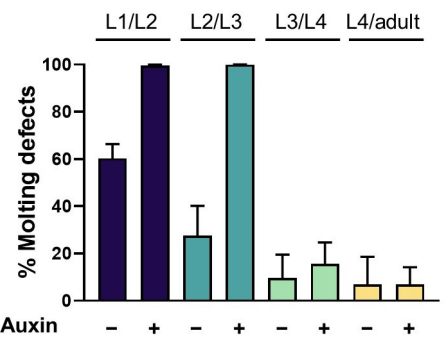

E

\section{N2 (Control)}

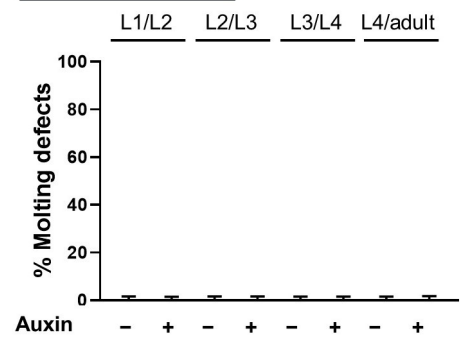

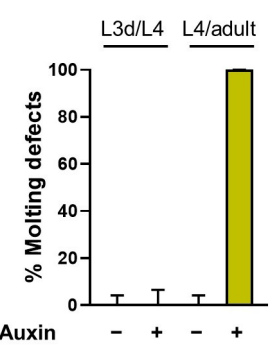

Auxin
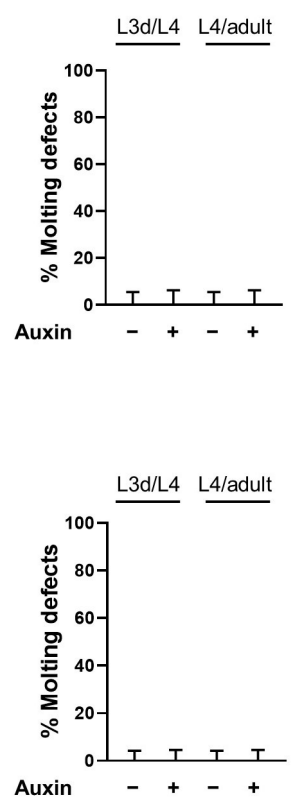

Figure 6. Requirements for NEKL kinases vary for different molts. (A) Schematic representation showing stages of larval development that were subjected to auxin-induced degradation of NEKLs (see Materials and Methods). (B) Schematic illustration showing how auxin-mediated degradation of NEKLs occurs. In the presence of auxin, TIR1-SCF complex binds to the AID tag and this interaction leads to ubiquitin-mediated degradation of NEKL::AID proteins. (C-E) Bar plots showing the percentage of moltingdefective worms during continuous (left) and dauer-interrupted (right) development in the presence and absence of auxin in nekl-2::aid (C), nekl3::aid (D), and wild-type (N2) (E) worms. Error bars represent 95\% confidence intervals. For additional details see Materials and Methods. 
exhibited molting defects at later developmental stages (Figures 6D). This indicates that the functional requirement for NEKL-3 during molting is minimal after entry into the L3 stage. In contrast, treatment of wild-type control worms with auxin did not lead to growth arrest at any stage (Figure 6E). We also note that a substantial proportion of NEKL-3::AID worms underwent arrest at $\mathrm{L} 1 \rightarrow \mathrm{L} 2$ and $\mathrm{L} 2 \rightarrow \mathrm{L} 3$ molts in the absence of auxin treatment (Figure 6D). This is likely due to the inhibitory effects of the AID tag on NEKL-3 as well as some activity of the TIR1 ubiquitin ligase in the absence of auxin (Joseph et al., 2020; Lambrus et al., 2018; Natsume et al., 2016). Our data also suggest that neither NEKL-2 nor NEKL-3 is required for the $L 3 d \rightarrow L 4$ molt during the dauer exit process but that NEKL-2 is required for the post-dauer $\mathrm{L} 4 \rightarrow$ adult molt (Figure 6C-D). As previously discussed, however, both NEKL-2 and NEKL-3 do appear to be necessary for the $L 2 d \rightarrow$ dauer molt. These findings indicate distinct NEKL requirements at specific molts, which may reflect different requirements for endocytosis at different larval stages.

The dauer developmental trajectory is unlikely to mitigate trafficking defects in nekl mutants We previously reported that NEKLs are required for epidermal membrane trafficking and that depletion of NEKLs leads to the reduced internalization of the epidermal cargo LRP-1 (Joseph et al., 2020). Moreover, we identified strong genetic suppressors of nekl molting defects that are directly involved in clathrin-mediated endocytosis including mutations affecting AP2 clathrinadaptor subunits as well as the AP2 allosteric activator FCHO-1 (Joseph et al., 2020). Importantly, these trafficking-related suppressors reduce both nekl-associated molting defects as well as defects in clathrin mobility and LRP-1 internalization (Joseph et al., 2020). To determine whether L2d affects nek/ trafficking deficits, we examined the localization of LRP-1 on the apical surface of hyp7 in daf-2(e1370); nekl-3::aid post-dauer adults. We found no evidence that passage through $\mathrm{L} 2 \mathrm{~d}$ and dauer can correct trafficking defects in NEKL-3 depleted adults, suggesting that suppression by L2d occurs through a distinct mechanism (Figure S3). This distinction between trafficking-related suppressors and $L 2 d$ is consistent with our observation that whereas loss of AP2 and FCHO-1 activity can suppress strong loss-of-function mutations in nekl-2 and nekl-3 (Joseph et al., 2020), transit through L2d does not. We note that experimental 
constraints allowed us to cleanly follow LRP-1 localization in adult worms only, and, although unlikely, it remains possible that L2d may affect LRP-1 trafficking specifically at this stage.

A
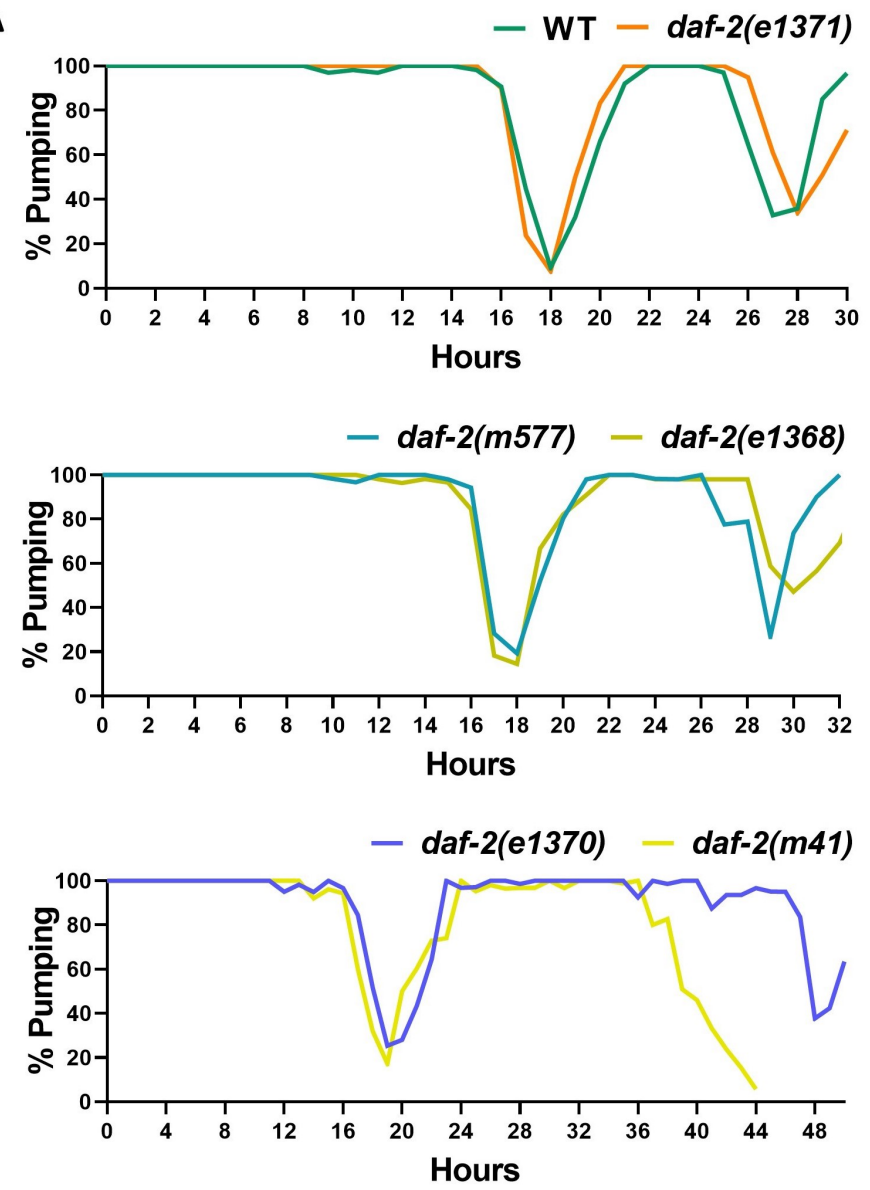

B

\section{Length of daf-2 L1 and L2 larval stages}

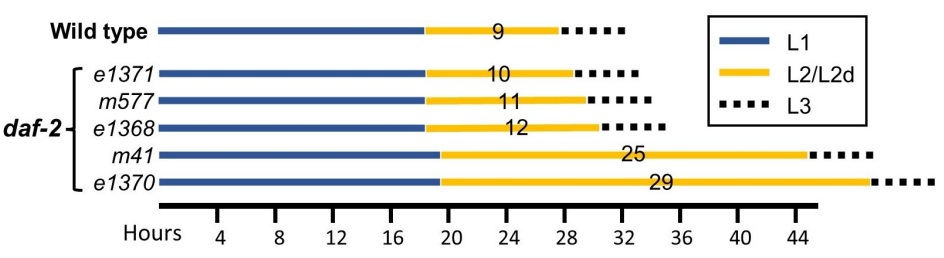

C

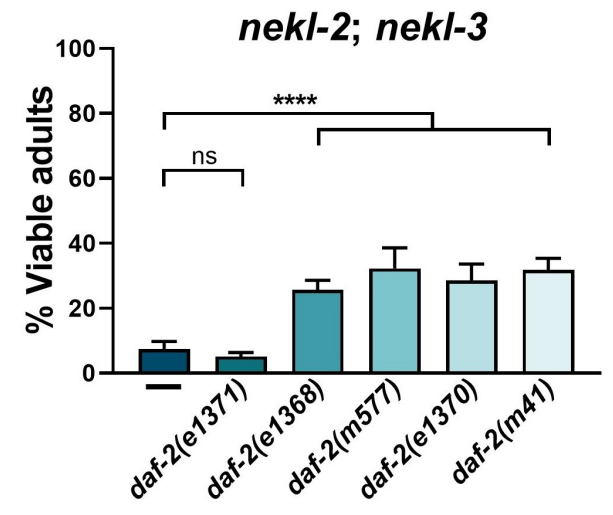

Figure 7. L2d length does not correlate with suppression by daf-2 alleles. A. Pharyngeal pumping in daf- 2 alleles was used to determine the length of $L 1$ and $L 2$ larval stages. Experimental details are described in Materials and Methods. (B) Summary of data from panel A. (C) Bar graphs showing viable adults (\%) scored in different daf-2 mutant backgrounds. Error bars represent $95 \%$ confidence intervals. Statistical significance was determined by Fisher's exact test; ${ }^{* * * *} \mathrm{p}$ $<0.0001$. ns, not significant. 


\section{The length of the second larval stage cannot account for suppression by L2d}

L2 diapause has a characteristic extended intermolt period as compared with the rapid development of an L2 larva after its first molt (Gems et al., 1998; Karp, 2018). We hypothesized that the longer intermolt period of an L2d larva may provide an extended window for nekl-2; nekl-3 mutants to complete processes required for successful molting. To determine whether the length of the L2d larval stage is important for the suppression of molting defects, we tested five alleles of daf-2-e1371, $m 577, e 1368, m 41$, and $e 1370$ - that have been extensively characterized and that differ in their phenotypic severity (Gems et al., 1998; Ruaud et al., 2011). Using a pharyngeal pumping assay to measure the characteristic pauses indicative of the lethargus phase of molting (Lažetić \& Fay, 2017b), we observed that the length of the second larval stage varied considerably among worms containing different daf-2 alleles (Figure 7A-B). Namely, the length of the L2 stage in alleles $e 1371, m 577$, and $e 1368$ was not substantially longer than that in wild type (10-12 h versus $9 \mathrm{~h}$, respectively), whereas alleles $m 41$ and $e 1370$ took $\sim 2-3$ times longer than wild type to complete the L2 stage (Figure 7A-B). Interestingly, all alleles except for e1371 suppressed nekl-2; nekl-3 mutants to a similar extent (Figure 7C), demonstrating that nekl suppression does not correlate with $\mathrm{L} 2 \mathrm{~d}$ length. These results indicate that the longer L2 inter-molt stage is unlikely to explain the observed suppression of molting defects by L2d.

\section{NEKLs are required for the upregulation of cyclically expressed molting genes}

Genetic studies have shown that steroid hormone signaling controls the activation of conserved nuclear hormone receptors, such as NHR-23 and NHR-25, which in turn regulate the expression of genes required for molting (Asahina et al., 2000; Kostrouchova et al., 2001; Kurzchalia \& Ward, 2003; Lažetić \& Fay, 2017b; Monsalve \& Frand, 2012). Correspondingly, developmental time-course transcriptome analyses have demonstrated that thousands of genes in C. elegans oscillate during each larval stage and that the relative timing of their expression is consistent between molts (Hendriks et al., 2014; Meeuse et al., 2020; Turek \& Bringmann, 2014). To better understand how NEKLs impact the molting process, we assessed the expression of four cyclically expressed genes ( $m / t-10$, nas-37, qua-1, and $f b n-1)$ that are required for molting in 
wild type and in nekl mutant backgrounds (Davis et al., 2004; Frand et al., 2005; Hao et al., 2006; Hendriks et al., 2014; Meeuse et al., 2020). To follow oscillating expression patterns, we used strains containing promoter fusions for each gene, with the promoter of interest fused to GFP tagged with the PEST sequence, which confers rapid degradation of the fluorescent marker (Frand et al., 2005; Hao et al., 2006; Meeuse et al., 2020).

As expected, wild-type synchronized larvae showed an oscillating pattern in accordance with previously published studies: $\mathrm{P}_{f b n-1}:: G F P-P E S T$ and $\mathrm{P}_{m / t-10:: G F P-P E S T}$ showed earliest and latest

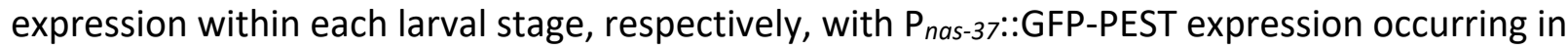
the interval (Figure 8A-C and Figure S4A) (Hendriks et al., 2014; Meeuse et al., 2020; Turek \& Bringmann, 2014). Notably, null mutations in nekl-2 and nekl-3 showed a dramatic reduction in the percentage of worms expressing these reporters during the L1 stage, as well as an $\sim 2$-fold increase in the length of time before expression was first detected (Figure 8A-C and Figure S5S6). For example, the first pulse of $\mathrm{P}_{m / t-10:: G F P-P E S T}$ expression was detected in wild-type $\mathrm{L1}$ larvae at $\sim 16$ hours, whereas in null nekl backgrounds expression was detectable only after $\sim 32$ hours and, unlike in wild-type larvae where it is expressed broadly, $\mathrm{P}_{m / t-10}:$ :GFP-PEST was confined to a small region in the anterior in mutants (Figure $8 \mathrm{~A}$ and Figure S5). We also tested a fourth molting reporter, $\mathrm{P}_{\text {qua-1 }}:: G F P:: H 2 B-P E S T$, in null mutants and observed a substantial delay in L1 expression (Figure S4B-C).

In the case of nekl-2; nekl-3 mutants, which arrest predominantly at the $\mathrm{L} 2 \rightarrow \mathrm{L} 3$ molt, we observed a slightly delayed but coherent peak of expression for $\mathrm{P}_{m / t-10}$ ::GFP-PEST during the L1 stage, followed by a less synchronous and delayed peak during the L2 stage (Figure 8A and Figure S5). We note that despite showing low/variable expression of $\mathrm{P}_{m / t-10}:$ :GFP-PEST for the remainder of the time course, these nekl-2; nekl-3 animals remained arrested at the $\mathrm{L} 2 \rightarrow \mathrm{L} 3$ molt. Likewise, nekl-2; nekl-3 strains containing $\mathrm{P}_{f b n-1}::$ GFP-PEST and $\mathrm{P}_{n a s-37:: G F P-P E S T}$ markers also exhibited a slight delay in L1 expression but, more importantly, displayed highly variable and asynchronous expression of these markers at the $L 2$ stage and in subsequent $L 2 \rightarrow L 3$ arrested larvae (Figure $8 \mathrm{~B}-\mathrm{C}$ ). We note that although the time course of $\mathrm{P}_{f b n-1}::$ GFP-PEST and 
A
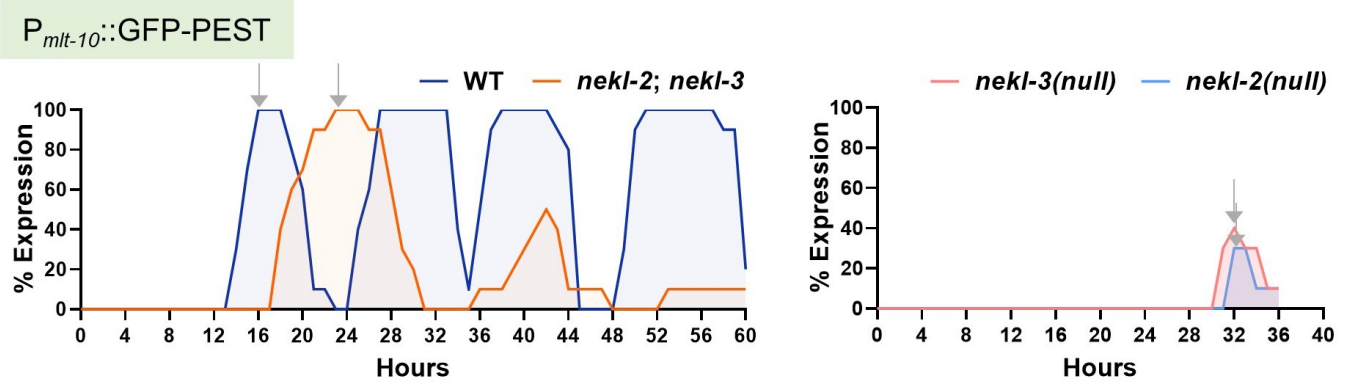

B
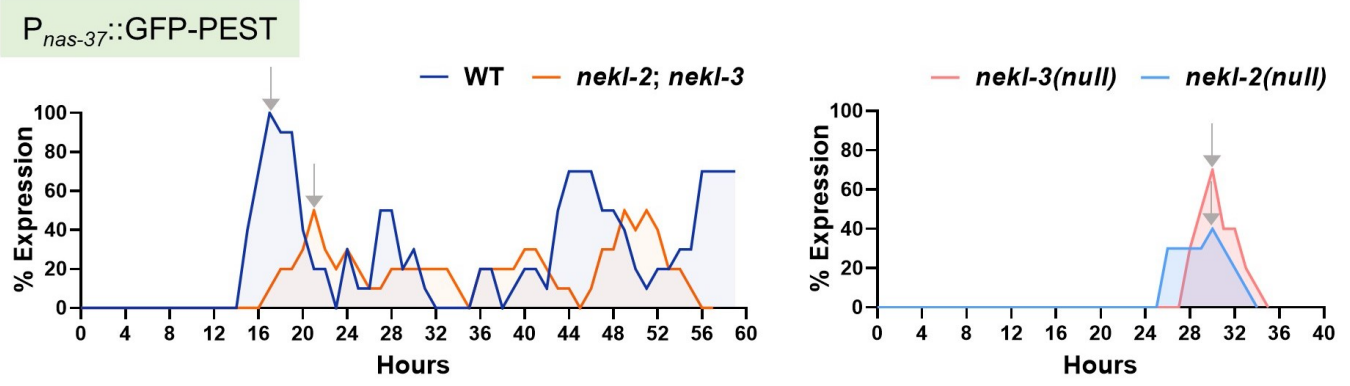

C

\section{$\mathrm{P}_{f b n-1:: G F P-P E S T}$}
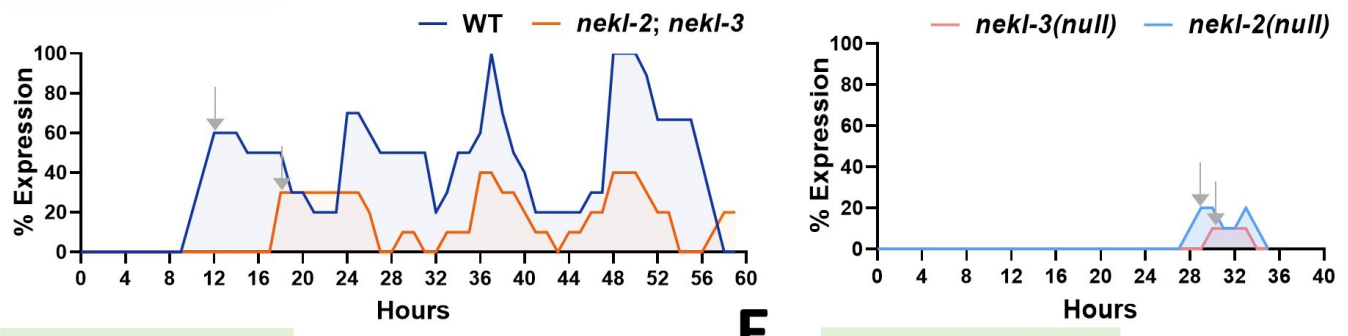

D

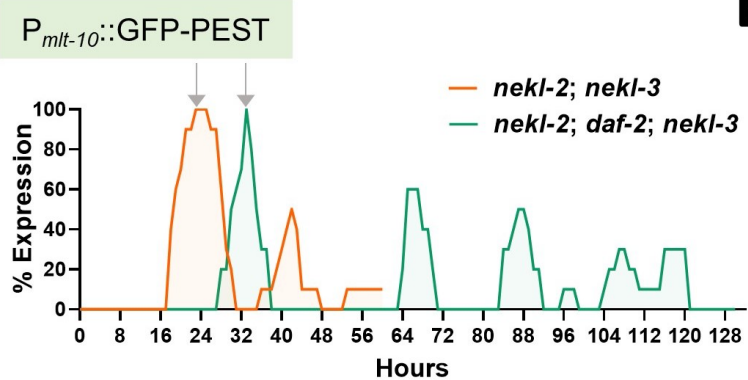

E $\quad P_{\text {nas-37::GFP-PEST }}$

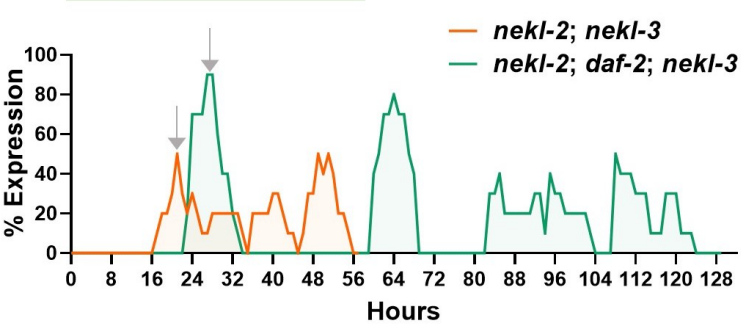

Figure 8. NEKLs are required for the synchronous and timely expression of cyclically expressed molting genes.

$(A-C)$ The percentage of worms expressing $P_{\text {mlt-10::GFP-PEST }}(\mathrm{A}), \mathrm{P}_{\text {nas }-37:: G F P-P E S T}(\mathrm{~B})$, and $\mathrm{P}_{\text {fbn-1 }}::$ GFP-PEST (C) was scored every hour (present or absent) after release from L1 arrest in synchronized cultures. Expression was observed in wild type, nekl-2; nekl-3 double mutants, and null nekl-2(gk839) and null nekl-3(gk506) mutant

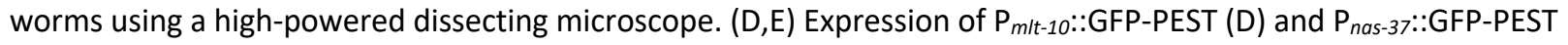
(E) was followed in nekl-2; nekl-3 mutants containing the daf-2(e1370) allele. In each experiment (A-E), at least 10 worms were scored every hour at $20^{\circ} \mathrm{C}$ for expression of the reporter. Grey arrow indicates peak expression of different reporters during late L1 stage.

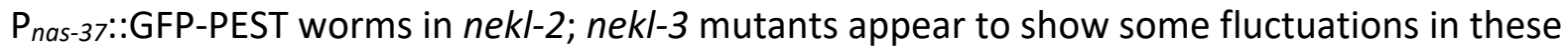
markers, these worms remained arrested at the $\mathrm{L} 2 \rightarrow \mathrm{L} 3$ molt. 
We also examined $\mathrm{P}_{m l t-10:: G F P-P E S T}$ in nekl-2(fd91) and nekl-3(sv3) mutants, which also arrest at

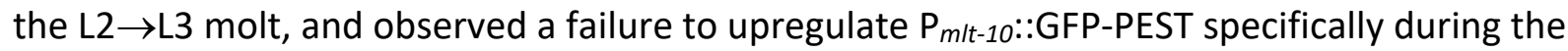
second larval stage (Figure S4D). Moreover, the ability of nekl-2(fd91) and nekl-3(sv3) to activate $\mathrm{P}_{m / t-10}:$ :GFP-PEST during $\mathrm{L} 2$ was reduced as compared with nekl-2; nekl-3 strains, consistent with these alleles causing a more penetrant molting defect than the double mutant (Lažetić \& Fay, 2017a; Yochem et al., 2015). Collectively, these observations demonstrate a strong correlation between the specific molting phenotypes of different nekl alleles and corresponding stage-specific defects in the expression of molting genes. Moreover, these results suggest that molting defects in nekl mutant backgrounds may be due to the reduced or delayed expression of genes required for the molting process.

Given the above observations, we were curious to know if the delays observed in the timing of the expression of molting genes in nekl mutants were specific to the molting process or whether other developmental processes might also be delayed in nekl mutants. To assess this, we made use of the $Q$ cell system, which undergoes a stereotypical pattern of cell divisions, migrations, and differentiation into neurons during the L1 stage (Figure S7; Middelkoop \& Korswagen, 2014). Based on previously defined stages, we scored Q cell developmental progression in wild-type, nekl-2 null, and nekl-3 null L1 larvae. Notably, we observed strong delays throughout $Q$ cell development, with final differentiation into neurons taking $\sim 1.5$-fold longer in nekl mutants than in wild type. These results suggest that reduced NEKL function not only delays the onset of molting gene expression but also slows the overall rate of development in these animals.

\section{nekl-2; nekl-3 transcriptional defects are bypassed by progression through L2d}

Our above results indicate that defects in the expression of molting genes during the second larval stage may account for the $\mathrm{L} 2 \rightarrow \mathrm{L} 3$ larval-arrest phenotype of nekl-2; nekl-3 mutants. Given that L2d can suppress nekl-2; nekl-3 molting defects, we were interested to determine whether transit through the $\mathrm{L} 2 \mathrm{~d} \rightarrow \mathrm{L} 3$ program also rescues expression defects in nekl-2; nekl-3 
mutants. Consistent with the suppression of molting defects, we observed clear and

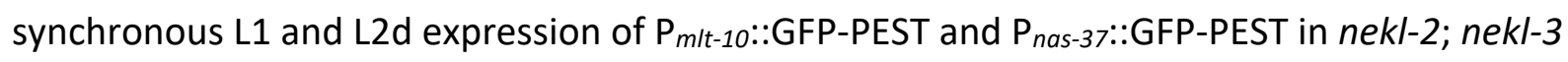
larvae that were shunted through $\mathrm{L} 2 \mathrm{~d} \rightarrow \mathrm{L} 3$ using a daf-2 loss-of-function mutation (Figure 8DE). Furthermore, these animals showed coherent expression peaks at the $\mathrm{L} 3 \rightarrow \mathrm{L} 4$ and $\mathrm{L} 4 \rightarrow$ adult transitions, consistent with progression through the remainder of larval development. We note, however, that the daf-2 mutation did not reverse delays in molting gene expression and, if anything, exacerbated this effect (Figure 8D-E). More broadly, we conclude that passage through $\mathrm{L} 2 \mathrm{~d} \rightarrow \mathrm{L} 3$ can sufficiently overcome transcriptional defects in nekl-2; nekl-3 mutants, leading to the suppression of molting defects.

\section{Discussion}

\section{Different requirements for NEKLs at different molts}

In this study, we showed that developmental choices can affect molting outcomes in C. elegans. We found that starvation, exposure to dauer pheromone, and Daf-c mutations-conditions that induce $\mathrm{L} 2 \mathrm{~d}-$ all led to suppression of $\mathrm{L} 2 \rightarrow \mathrm{L} 3$ molting defects in nekl-2; nekl-3 mutants.

Starvation and dauer formation modulate other developmental processes in C. elegans, such as vulval induction (Battu et al., 2003; Baugh \& Hu, 2020; Ferguson \& Horvitz, 1985). In addition, passage through L2d and dauer corrects heterochronic defects (Abrahante et al., 2003;

Ferguson \& Horvitz, 1985; Liu \& Ambros, 1991), in part by rewiring the gene regulatory network that controls temporal patterning (Ilbay \& Ambros, 2019). In contrast, our findings suggest that the observed suppression of molting defects occurs because of different requirements for NEKL activities at different molts.

Figure 9A depicts a summary of our findings for NEKL-2 and NEKL-3 requirements at each molt. For example, timed-inactivation studies using NEKL-tagged AID strains revealed requirements for NEKL-2 and NEKL-3 at both the $\mathrm{L} 1 \rightarrow \mathrm{L} 2$ and $\mathrm{L} 2 \rightarrow \mathrm{L} 3$ molts, whereas only NEKL-2 is required for progression through the $\mathrm{L} 3 \rightarrow \mathrm{L} 4$ and $\mathrm{L} 4 \rightarrow$ adult molts. In addition, the inability of partial lossof-function alleles of nek/s to produce dauers under starvation conditions that induce $L 2 d$, indicates a requirement for both NEKL-2 and NEKL-3 in the L2d $\rightarrow$ dauer molt. Depleting NEKLs in dauer larvae and then moving those larvae to plates with bacteria also suggests that neither 
NEKL-2 nor NEKL-3 is required for the $L 3 d \rightarrow$ post-dauer $L 4$ molt, although NEKL-2 is still required for the post-dauer $L 4 \rightarrow$ post-dauer adult molt.

In addition, genetic data which are summarized in Figure 9B, indicate that there is a greater requirement for NEKL-2 and NEKL-3 in the $L 2 \rightarrow L 3$ molt than in the $L 1 \rightarrow L 2$ molt. Specifically, nekl-2; nekl-3 double mutants, along with nekl-3(sv3) and nekl-2(fd91) single mutants, exhibited molting defects at the $\mathrm{L} 2 \rightarrow \mathrm{L} 3$ molt, indicating that they were able to successfully transit the $\mathrm{L} 1 \rightarrow \mathrm{L} 2$ molt despite the partial reduction in NEKL activity. This observation also correlates with the expression of molting genes in these nekl mutant backgrounds, which showed a slightly delayed but nevertheless clear upregulation of molting genes during L1 but a failure to consistently upregulate these genes during L2. Lastly, the ability of L2d to suppress molting defects in nekl-2; nekl-3 double mutants indicates that NEKL requirements are lower for the $\mathrm{L} 2 \mathrm{~d} \rightarrow \mathrm{L} 3$ transition than for $\mathrm{L} 2 \rightarrow \mathrm{L} 3$. Our studies also provide an explanation for how nekl-2; nekl-3 mutants can successfully transverse the $\mathrm{L} 3 \rightarrow \mathrm{L} 4$ and $\mathrm{L} 4 \rightarrow$ adult molts after completing $\mathrm{L} 2 \mathrm{~d} \rightarrow \mathrm{L3}$. Namely, given that NEKL-3 is not required for the $\mathrm{L} 3 \rightarrow \mathrm{L} 4$ or $\mathrm{L} 4 \rightarrow$ adult molts, the weak (aphenotypic) loss-of-function allele of nekl-2, fd81, would not be expected to cause arrest at these stages.

\section{The role of NEKLs in trafficking, transcription, and molting}

By promoting the cyclic expression of hundreds of molting-related genes, steroid hormone signaling is a central feature of larval development. Likewise, the uptake of steroid hormone precursors, such as cholesterol, is essential for steroid hormone signaling. One such receptor, LRP-1, mediates the internalization of cholesterol by the epidermis and is required specifically in hyp7 for the completion of molting (Yochem et al., 1999). We previously showed that NEKL-2 and NEKL-3 regulate clathrin-mediated endocytosis and that depletion of NEKLs leads to the impaired endocytosis of LRP-1 at the apical membrane (Joseph et al., 2020). In the current study, we found that the expression of cyclically regulated molting genes is misregulated in nekl mutants and that these expression defects correspond closely with the strength of nekl alleles and with the timing of their developmental arrest points. Collectively, these findings imply that loss of NEKLs impairs steroid hormone signaling and thus the ability of larvae to transcribe 


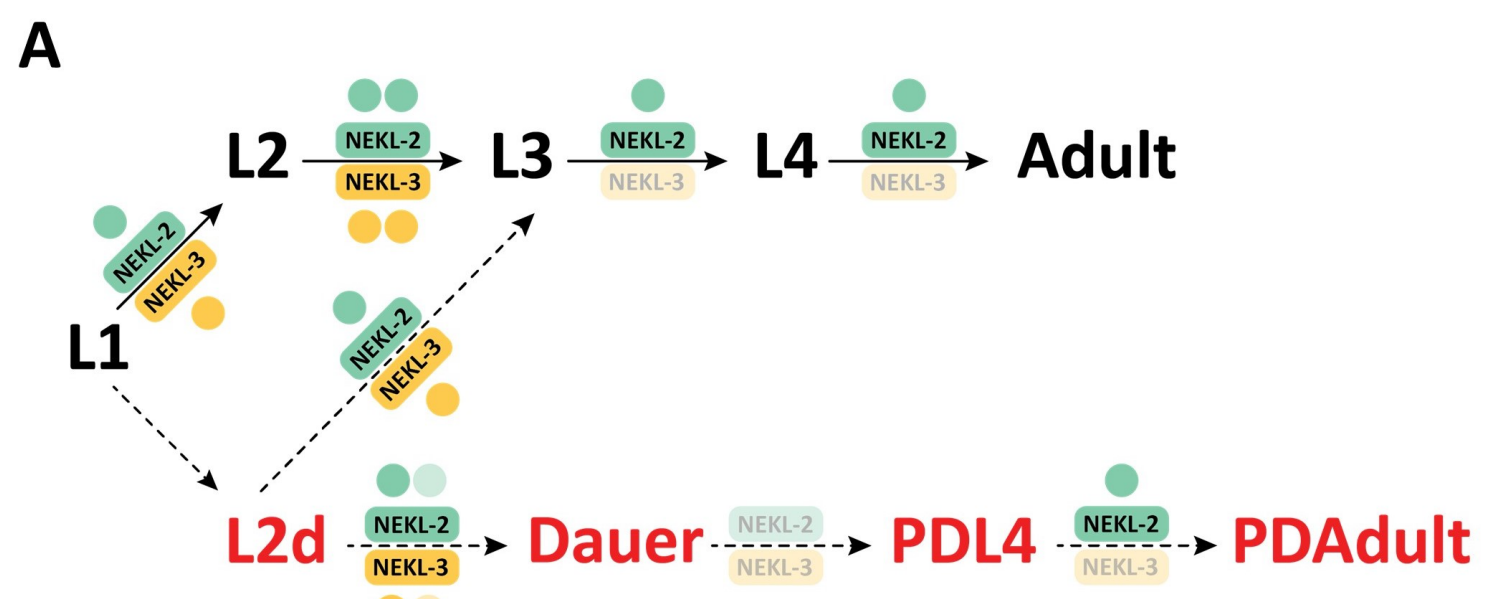

B

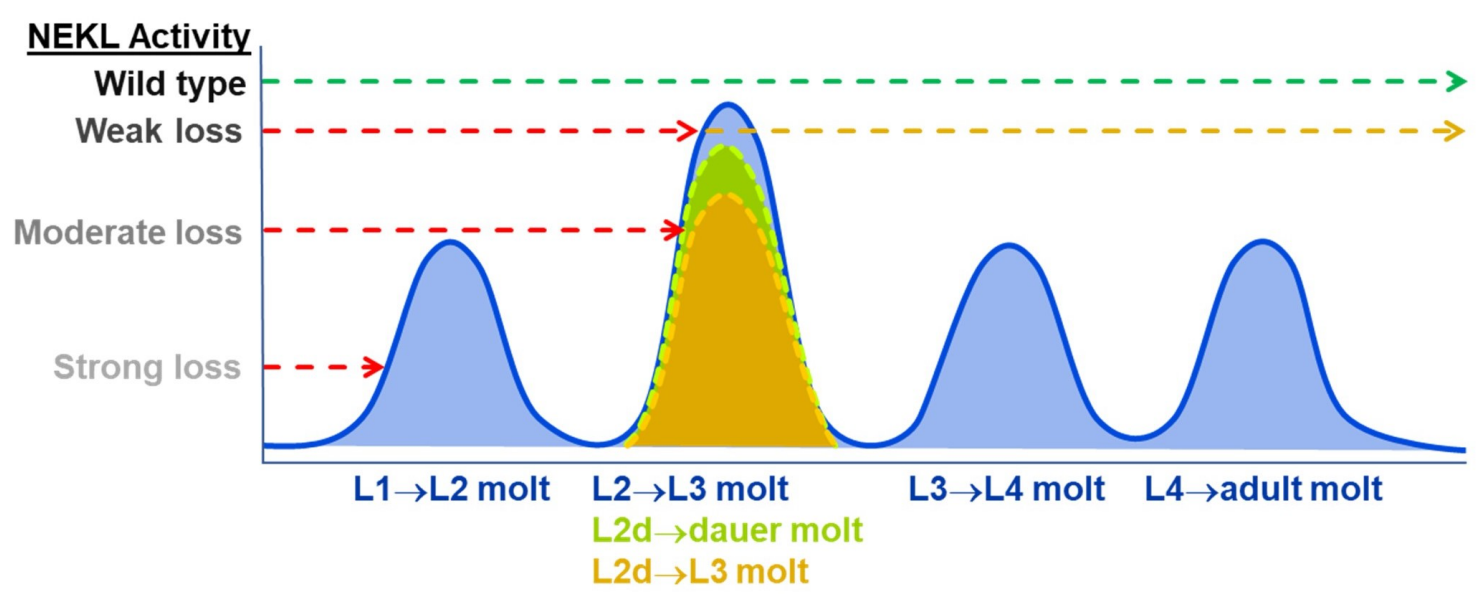

Figure 9. Summary of NEKL requirements at different molts. (A) Whereas NEKL-2 was required for all four larval molts, NEKL-3 was primarily required for the $\mathrm{L} 1 \rightarrow \mathrm{L} 2$ and $\mathrm{L} 2 \rightarrow \mathrm{L} 3$ molts. The requirement for NEKL kinases during the $\mathrm{L} 2 \rightarrow \mathrm{L} 3$ molt is higher (two dots) than during the $\mathrm{L} 1 \rightarrow \mathrm{L} 2, \mathrm{~L} 2 \mathrm{~d} \rightarrow \mathrm{L} 3$ or $\mathrm{L} 2 \mathrm{~d} \rightarrow$ dauer molts (fewer than two dots). Neither NEKL-2 nor NEKL-3 appears to be required for the molt immediately following dauer exit (dauer $\rightarrow$ L4). The requirements for NEKL-2 and NEKL-3 during the final molt to adulthood appear similar for worms under continuous growth conditions or post dauer. PD, post-dauer. (B) A graphical representation showing threshold requirements for NEKL kinase activities at different molts. Whereas weak loss-of-function nekl-2; nekl-3 mutants arrested at the $\mathrm{L} 2 \rightarrow \mathrm{L} 3$ molt, they were able to transit the $\mathrm{L} 2 \mathrm{~d} \rightarrow \mathrm{L} 3$ molt and, in the case of ascaroside treatment, the $\mathrm{L} 2 \mathrm{~d} \rightarrow$ dauer molt, indicating that these latter two transitions have a lower threshold requirement for NEKL kinases. Strong reduction in kinase activity in intermediate loss-of-function mutants [nekl-2(fd91) and nekl-3(sv3)] was still sufficient to complete the L1 $\rightarrow \mathrm{L2}$ molt, however, these mutants were unable to complete either $\mathrm{L} 2 \rightarrow \mathrm{L} 3$ or $\mathrm{L} 2 \mathrm{~d} \rightarrow \mathrm{L} 3$ molt. Finally, complete loss of kinase activity in null mutants [nekl-2(gk839) and nekl-3(gk506)] led to arrest at the L1 $\rightarrow$ L2 molt. 
In this study we found that transit through the uncommitted $L 2 d$ stage $(L 2 d \rightarrow L 3)$ can suppress both molting and transcriptional defects in nekl-2; nekl-3 mutants, indicating that developmental plasticity and environmental context can affect this phenotype. Experiments suggest that $\mathrm{L} 2 \mathrm{~d}$ suppression is not due to either a correction of trafficking defects or an increase in the length of this larval stage. Rather, we infer that different stages of larval development may have distinct requirements for the level of steroid hormones and that the threshold for certain transitions, such as $L 1 \rightarrow L 2$ and $L 2 d \rightarrow L 3$, may be less than that for other transitions, such as $L 2 \rightarrow L 3$. Alternatively, it is possible that during certain life stages a sufficient level of steroid-hormone building blocks is naturally present, such that there is less dependence on importing additional signaling precursors via NEKLs and epidermal endocytosis. Consistent with this possibility, neither NEKL-2 nor NEKL-3 was required for the dauer $\rightarrow$ L4 transition, and dauer larvae are known to concentrate lipids (Jeong et al., 2005; Popham \& Webster, 1979). Future studies will seek to understand precisely how NEKL kinases regulate targets involved in intracellular trafficking and will increase our knowledge of which epidermal trafficking cargoes are critical for molting.

\section{Acknowledgments}

We thank Amy Fluet for editing this manuscript and Eric Jorgenson, Paul Sternberg, Alison Frand, and Helge Grosshans for strains. This project was supported by R35 GM136236 to DSF and by an Institutional Development Award (IDeA) from the National Institute of General Medical Sciences of the National Institutes of Health (P20GM103432). 


\section{References}

Abrahante, J. E., Daul, A. L., Li, M., Volk, M. L., Tennessen, J. M., Miller, E. A., \& Rougvie, A. E. (2003). The Caenorhabditis elegans hunchback-like gene lin-57/hbl-1 controls developmental time and is regulated by microRNAs. Developmental Cell, 4(5), 625-637. https://doi.org/10.1016/S1534-5807(03)00127-8

Antebi, A. (2015). Nuclear receptor signal transduction in C. elegans. WormBook : The Online Review of C. Elegans Biology, 1-49. https://doi.org/10.1895/wormbook.1.64.2

Arribere, J. A., Bell, R. T., Fu, B. X. H., Artiles, K. L., Hartman, P. S., \& Fire, A. Z. (2014). Efficient marker-free recovery of custom genetic modifications with CRISPR/Cas9 in caenorhabditis elegans. Genetics, 198(3), 837-846. https://doi.org/10.1534/genetics.114.169730

Asahina, M., Ishihara, T., Jindra, M., Kohara, Y., Katsura, I., \& Hirose, S. (2000). The conserved nuclear receptor Ftz-F1 is required for embryogenesis, moulting and reproduction in Caenorhabditis elegans. Genes to Cells, 5(9), 711-723. https://doi.org/10.1046/j.13652443.2000.00361.x

Battu, G., Hoier, E. F., \& Hajnal, A. (2003). The C. elegans G-protein-coupled receptor SRA-13 inhibits RAS/MAPK signalling during olfaction and vulval development. Development, 130(12), 2567-2577. https://doi.org/10.1242/dev.00497

Baugh, L. R., \& Hu, P. J. (2020). Starvation responses throughout the caenorhabditis elegans life cycle. In Genetics (Vol. 216, Issue 4). https://doi.org/10.1534/genetics.120.303565

Butcher, R. A., Ragains, J. R., Kim, E., \& Clardy, J. (2008). A potent dauer pheromone component in Caenorhabditis elegans that acts synergistically with other components. Proceedings of the National Academy of Sciences of the United States of America, 105(38), 14288-14292. https://doi.org/10.1073/pnas.0806676105

Cassada, R. C., \& Russell, R. L. (1975). The dauerlarva, a post-embryonic developmental variant of the nematode Caenorhabditis elegans. Developmental Biology, 46(2), 326-342. https://doi.org/10.1016/0012-1606(75)90109-8

Davis, M. W., Birnie, A. J., Chan, A. C., Page, A. P., \& Jorgensen, E. M. (2004). A conserved metalloprotease mediates ecdysis in Caenorhabditis elegans. Development, 131(23), 60016008. https://doi.org/10.1242/dev.01454 
Ferguson, E. L., \& Horvitz, H. R. (1985). Identification and characterization of 22 genes that affect the vulval cell lineages of the nematode Caenorhabditis elegans. Genetics, 110(1), 17-72. https://doi.org/10.1093/genetics/110.1.17

Fielenbach, N., \& Antebi, A. (2008). C. elegans dauer formation and the molecular basis of plasticity. Genes and Development, 22(16), 2149-2165. https://doi.org/10.1101/gad.1701508

Frand, A. R., Russel, S., \& Ruvkun, G. (2005). Functional genomic analysis of c. elegans molting. PLoS Biology, 3(10). https://doi.org/10.1371/journal.pbio.0030312

Gems, D., Sutton, A. J., Sundermeyer, M. L., Albert, P. S., King, K. V., Edgley, M. L., Larsen, P. L., \& Riddle, D. L. (1998). Two pleiotropic classes of daf-2 mutation affect larval arrest, adult behavior, reproduction and longevity in Caenorhabditis elegans. Genetics, 150(1), 129155.

Golden, J. W., \& Riddle, D. L. (1984a). A pheromone-induced developmental switch in Caenorhabditis elegans: Temperature-sensitive mutants reveal a wild-type temperaturedependent process. Proceedings of the National Academy of Sciences of the United States of America, 81(3), 819-823. https://doi.org/10.1073/pnas.81.3.819

Golden, J. W., \& Riddle, D. L. (1984b). The Caenorhabditis elegans dauer larva: Developmental effects of pheromone, food, and temperature. Developmental Biology, 102(2), 368-378. https://doi.org/10.1016/0012-1606(84)90201-X

Gumienny, T. L., \& Savage-Dunn, C. (2013). TGF- $\beta$ signaling in C. elegans. WormBook : The Online Review of C. Elegans Biology, 1-34. https://doi.org/10.1895/wormbook.1.22.2 Hall, S. E., Beverly, M., Russ, C., Nusbaum, C., \& Sengupta, P. (2010). A Cellular Memory of Developmental History Generates Phenotypic Diversity in C. elegans. Current Biology, 20(2), 149-155. https://doi.org/10.1016/j.cub.2009.11.035

Hao, L., Mukherjee, K., Liegeois, S., Baillie, D., Labouesse, M., \& Bürglin, T. R. (2006). The hedgehog-related gene qua-1 is required for molting in Caenorhabditis elegans. Developmental Dynamics, 235(6), 1469-1481. https://doi.org/10.1002/dvdy.20721 Hendriks, G. J., Gaidatzis, D., Aeschimann, F., \& Großhans, H. (2014). Extensive Oscillatory Gene Expression during C.elegans Larval Development. Molecular Cell, 53(3), 380-392. 
https://doi.org/10.1016/j.molcel.2013.12.013

Holland, A. J., Fachinetti, D., Han, J. S., \& Cleveland, D. W. (2012). Inducible, reversible system

for the rapid and complete degradation of proteins in mammalian cells. Proceedings of the National Academy of Sciences of the United States of America, 109(49).

https://doi.org/10.1073/pnas.1216880109

Hollister, K. A., Conner, E. S., Zhang, X., Spell, M., Bernard, G. M., Patel, P., De Carvalho, A. C. G. V., Butcher, R. A., \& Ragains, J. R. (2013). Ascaroside activity in Caenorhabditis elegans is highly dependent on chemical structure. Bioorganic and Medicinal Chemistry, 21(18), 5754-5769. https://doi.org/10.1016/j.bmc.2013.07.018

Hu, P. J. (2007). Dauer. WormBook : The Online Review of C. Elegans Biology, 734, 1-19. https://doi.org/10.1895/wormbook.1.144.1

Ilbay, O., \& Ambros, V. (2019). Pheromones and Nutritional Signals Regulate the Developmental Reliance on let-7 Family MicroRNAs in C. elegans. Current Biology, 29(11), 1735-1745.e4. https://doi.org/10.1016/j.cub.2019.04.034

Jeong, P. Y., Jung, M., Yim, Y. H., Kim, H., Park, M., Hong, E., Lee, W., Kim, Y. H., Kim, K., \& Paik, Y. K. (2005). Chemical structure and biological activity of the Caenorhabditis elegans dauer-inducing pheromone. Nature, 433(7025), 541-545. https://doi.org/10.1038/nature03201

Joseph, B. B., Wang, Y., Edeen, P., Lazetic, V., Grant, B. D., \& Fay, D. S. (2020). Control of clathrin-mediated endocytosis by NIMA family kinases. In PLoS Genetics (Vol. 16, Issue 2). https://doi.org/10.1371/journal.pgen.1008633

Karp, X. (2018). Working with dauer larvae. WormBook, 1-19.

https://doi.org/10.1895/wormbook.1.180.1

Kim, H., Ishidate, T., Ghanta, K. S., Seth, M., Conte, D., Shirayama, M., \& Mello, C. C. (2014). A Co-CRISPR strategy for efficient genome editing in Caenorhabditis elegans. Genetics, 197(4), 1069-1080. https://doi.org/10.1534/genetics.114.166389

Kimura, K. D., Tissenbaum, H. A., Liu, Y., \& Ruvkun, G. (1997). Daf-2, an insulin receptor-like gene that regulates longevity and diapause in Caenorhabditis elegans. Science, 277(5328), 942-946. https://doi.org/10.1126/science.277.5328.942 
Kostrouchova, M., Krause, M., Kostrouch, Z., \& Rall, J. E. (2001). Nuclear hormone receptor CHR3 is a critical regulator of all four larval molts of the nematode Caenorhabditis elegans. 98(13), 7360-7365. www.pnas.orgcgidoi10.1073pnas.131171898

Kurzchalia, T. V., \& Ward, S. (2003). Why do worms need cholesterol? Nature Cell Biology, 5(8), 684-688. https://doi.org/10.1038/ncb0803-684

Lambrus, B. G., Moyer, T. C., \& Holland, A. J. (2018). Applying the auxin-inducible degradation system for rapid protein depletion in mammalian cells. Methods in Cell Biology, 144, 107135. https://doi.org/10.1016/BS.MCB.2018.03.004

Lažetić, V., \& Fay, D. S. (2017a). Conserved ankyrin repeat proteins and their NIMA kinase partners regulate extracellular matrix remodeling and intracellular trafficking in Caenorhabditis elegans. Genetics, 205(1), 273-293. https://doi.org/10.1534/genetics.116.194464

Lažetić, V., \& Fay, D. S. (2017b). Molting in C. elegans. Worm, 6(1), e1330246. https://doi.org/10.1080/21624054.2017.1330246

Lee, R. Y. N., Hench, J., \& Ruvkun, G. (2001). Regulation of C. elegans DAF-16 and its human ortholog FKHRL1 by the daf-2 insulin-like signaling pathway. Current Biology, 11(24), 19501957. https://doi.org/10.1016/S0960-9822(01)00595-4

Li, C. H., \& Tam, P. K. S. (1998). An iterative algorithm for minimum cross entropy thresholding. Pattern Recognition Letters, 19(8), 771-776. https://doi.org/10.1016/S01678655(98)00057-9

Lin, K. (1997). daf-16: An HNF-3/forkhead Family Member That Can Function to Double the LifeSpan of Caenorhabditis elegans. Science, 278(5341), 1319-1322.

https://doi.org/10.1126/science.278.5341.1319

Lin, Kui, Hsin, H., Libina, N., \& Kenyon, C. (2001). Regulation of the Caenorhabditis elegans longevity protein DAF-16 by insulin/IGF-1 and germline signaling. Nature Genetics, 28(2), 139-145. https://doi.org/10.1038/88850

Liu, Z., \& Ambros, V. (1991). Alternative temporal control systems for hypodermal cell differentiation in Caenorhabditis elegans. Nature, 350(6314), 162-165. https://doi.org/10.1038/350162a0 
Mao, Y., Han, S., Zhang, S., Zhang, Z., Kong, C., Chen, H., Li, Z., Cai, P., Han, B., \& Wang, L. (2020). An approach using Caenorhabditis elegans screening novel targets to suppress tumour cell proliferation. Cell Proliferation, 53(6). https://doi.org/10.1111/cpr.12832

Meeuse, M. W., Hauser, Y. P., Morales Moya, L. J., Hendriks, G., Eglinger, J., Bogaarts, G., Tsiairis, C., \& Großhans, H. (2020). Developmental function and state transitions of a gene expression oscillator in Caenorhabditis elegans. Molecular Systems Biology, 16(7), 1-21. https://doi.org/10.15252/msb.20209498

Middelkoop, T. C., \& Korswagen, H. C. (2014). Development and migration of the C. elegans Q neuroblasts and their descendants. WormBook, 1-23. https://doi.org/10.1895/wormbook.1.173.1

Monsalve, G. C., \& Frand, A. R. (2012). Toward a unified model of developmental timing. Worm, 1(4), 221-230. https://doi.org/10.4161/worm.20874

Natsume, T., Kiyomitsu, T., Saga, Y., \& Kanemaki, M. T. (2016). Rapid Protein Depletion in Human Cells by Auxin-Inducible Degron Tagging with Short Homology Donors. Cell Reports, 15(1), 210-218. https://doi.org/10.1016/j.celrep.2016.03.001

Ogg, S., Paradis, S., Gottlieb, S., Patterson, G. I., Lee, L., Tissenbaum, H. A., \& Ruvkun, G. (1997). TheForkhead transcription factorDAF-16 transduces insulin-likemetabolic and longevity signals in C.elegans Scott. Nature, 389(October).

Paix, A., Folkmann, A., Rasoloson, D., \& Seydoux, G. (2015). High Efficiency, Homology-Directed Genome Editing in Caenorhabditis elegans Using CRISPR-Cas9 Ribonucleoprotein Complexes. https://doi.org/10.1534/genetics.115.179382

Paix, A., Wang, Y., Smith, H. E., Lee, C. Y. S., Calidas, D., Lu, T., Smith, J., Schmidt, H., Krause, M. W., \& Seydoux, G. (2014). Scalable and versatile genome editing using linear DNAs with microhomology to Cas9 sites in Caenorhabditis elegans. Genetics, 198(4), 1347-1356. https://doi.org/10.1534/genetics.114.170423

Popham, J. D., \& Webster, J. M. (1979). Aspects of the fine structure of the dauer larva of the nematode caenorhabditis elegans. Canadian Journal of Zoology, 57(4), 794-800. https://doi.org/10.1139/z79-098

Porta-de-la-Riva, M., Fontrodona, L., Villanueva, A., \& Cerón, J. (2012). Basic Caenorhabditis 
elegans methods: Synchronization and observation. Journal of Visualized Experiments, 64, 1-9. https://doi.org/10.3791/4019

Ren, P., Lim, C. S., Johnsen, R., Albert, P. S., Pilgrim, D., \& Riddle, D. L. (1996). Control of C. elegans larval development by neuronal expression of a TGF- $\beta$ homolog. Science, 274(5291), 1389-1391. https://doi.org/10.1126/science.274.5291.1389

Riddle, D. L., \& Albert, P. S. (1997). Genetic and Environmental Regulation of Dauer Larva Development. In C. elegans II (Vol. 64, Issue 4). https://doi.org/10.1034/j.16000854.2002.30104.x

Ruaud, A. F., Katic, I., \& Bessereau, J. L. (2011). Insulin/insulin-like growth factor signaling controls non-dauer developmental speed in the nematode Caenorhabditis elegans. Genetics, 187(1), 337-343. https://doi.org/10.1534/genetics.110.123323

Schaedel, O. N., Gerisch, B., Antebi, A., \& Sternberg, P. W. (2012). Hormonal signal amplification mediates environmental conditions during development and controls an irreversible commitment to adulthood. PLoS Biology, 10(4), 1-18.

https://doi.org/10.1371/journal.pbio.1001306

Schindelin, J., Arganda-Carreras, I., Frise, E., Kaynig, V., Longair, M., Pietzsch, T., Preibisch, S., Rueden, C., Saalfeld, S., Schmid, B., Tinevez, J. Y., White, D. J., Hartenstein, V., Eliceiri, K., Tomancak, P., \& Cardona, A. (2012). Fiji: An open-source platform for biological-image analysis. Nature Methods, 9(7), 676-682. https://doi.org/10.1038/nmeth.2019

Shih, P. Y., Lee, J. S., \& Sternberg, P. W. (2019). Genetic markers enable the verification and manipulation of the dauer entry decision. Developmental Biology, 454(2), 170-180. https://doi.org/10.1016/j.ydbio.2019.06.009

Shim, Y. H., Chun, J. H., Lee, E. Y., \& Paik, Y. K. (2002). Role of cholesterol in germ-line development of Caenorhabditis elegans. Molecular Reproduction and Development, 61(3), 358-366. https://doi.org/10.1002/mrd.10099

Suzuki, Y., \& Nijhout, H. F. (2008). Genetic basis of adaptive evolution of a polyphenism by genetic accommodation. Journal of Evolutionary Biology, 21(1), 57-66. https://doi.org/10.1111/j.1420-9101.2007.01464.x

Turek, M., \& Bringmann, H. (2014). Gene expression changes of Caenorhabditis elegans larvae 
during molting and sleep-like lethargus. PLOS ONE, 9(11), 25-28.

https://doi.org/10.1371/journal.pone.0113269

Yochem, J., Lažetić, V., Bell, L., Chen, L., \& Fay, D. (2015). C. elegans NIMA-related kinases NEKL2 and NEKL-3 are required for the completion of molting. Developmental Biology, 398(2), 255-266. https://doi.org/10.1016/j.ydbio.2014.12.008

Yochem, J., Tuck, S., Greenwald, I., \& Han, M. (1999). A gp330/megalin-related protein is required in the major epidermis of Caenorhabditis elegans for completion of molting. Development, 126(3), 597-606. https://doi.org/10.1242/dev.126.3.597

Zhang, L., Ward, J. D., Cheng, Z., \& Dernburg, A. F. (2015). The auxin-inducible degradation (AID) system enables versatile conditional protein depletion in C. elegans. Development (Cambridge), 142(24), 4374-4384. https://doi.org/10.1242/dev.129635 